\title{
Simulation Algorithm of Typical Modulated Poisson-Voronoi Cells and Application to Telecommunication Network Modelling
}

\author{
F. Fleischer ${ }^{\dagger}, *$ C. Gloaguen ${ }^{\ddagger}$, H. Schmidt ${ }^{\dagger}$, \\ V. SCHMidT ${ }^{\S}$ and F. SCHWEIGGERT $\%$ \\ $\dagger^{\dagger}$ Medical Data Services, Biostatistics \\ Boehringer Ingelheim Pharma GmbH \& Co. KG \\ 88397 Biberach and 55216 Ingelheim, Germany \\ *E-mail: frank.fleischer@boehringer-ingelheim.com \\ $¥$ France Telecom Research and Development \\ 92794 Issy les Moulineaux Cedex 9, France \\ $\S$ Institute of Stochastics \\ University Ulm, 89069 Ulm, Germany \\ IInstitute of Applied Information Processing \\ University Ulm, 89069 Ulm, Germany
}

Received December 05, 2007

Revised May 06, 2008

\begin{abstract}
We consider modulated Poisson-Voronoi tessellations, intended as models for telecommunication networks on a nationwide scale. By introducing an algorithm for the simulation of the typical cell of the latter tessellation, we lay the mathematical foundation for such a global analysis. A modulated Poisson-Voronoi tessellation has an intensity which is spatially variable and, hence, is able to provide a broad spectrum of model scenarios. Nevertheless, the considered tessellation model is stationary and we consider the case where the modulation is generated by a Boolean germ-grain model with circular grains. These circular grains may either have a deterministic or random but bounded radius. Furthermore, based on the introduced simulation algorithm for the typical cell and on Neveu's exchange formula for Palm probability measures, we show how to estimate the mean distance from a randomly chosen location to its nearest Voronoi cell nucleus. The latter distance is interpreted as an important basic cost characteristic in telecommunication networks, especially for the computation of more sophisticated functionals later on. Said location is chosen at random among the points of another modulated Poisson process where the modulation is generated by the same Boolean model as for the nuclei. The case of a completely random placement for the considered location is thereby included as a special case. The estimation of the cost functional is performed in a way such that a simulation of the location placement is not necessary. Test methods for the correctness of the algorithm based on tests for random software are briefly discussed. Numerical examples are provided for characteristics of the typical cell as well as for the cost functional. We conclude with some remarks about extensions and modifications of the model regarded in this paper, like modulated Poisson-Delaunay tessellations.
\end{abstract}

Key words: stochastic geometry, telecommunication network modelling, Neveu's exchange formula, Voronoi tessellation, Boolean model

\section{Introduction}

During the last years spatial stochastic modelling of telecommunication networks has become an established alternative to more traditional economic approaches for cost measurement and strategic planning of telecommunication networks. While the geometric structure of such a model allows a more realistic view to location dependent network characteristics than conventional models, the random 
setting can reflect the network variability in time and space.

Among the examples where stochastic-geometric models have been considered recently are mobile telecommunication systems, multi-cast networks and switching networks all based on tools from stochastic geometry like modulated Poisson-Voronoi tessellations (see Błaszczyszyn and Schott [7], [8]), PoissonVoronoi aggregated tessellations (see Bacelli, Klein, Lebourges and Zuyev [3] and Thoumatchenko and Zuyev [19]), superpositions of Poisson-Voronoi tessellations (see Bacelli, Gloaguen and Zuyev [2]), spanning trees (see Bacelli, Kofman and Rougier [5] and Bacelli and Zuyev [6]), and coverage processes (see Bacelli and Błaszczyszyn [1]). The Stochastic Subscriber Line Model (SSLM), cf. [10] for example, is a particular example of a random-geometric approach to model networks with an explicit description of the underlying road system.

However, a key issue in modelling communication networks, especially from a global perspective, is the consideration of instationarities, in particular with respect to the underlying geometry of the network. If we regard Fig. 1 for example, we observe that the displayed network devices of two regions in France are scattered spatially with a varying intensity. More precisely the devices are scattered denser in urban areas than in the rural landscape, due to the fact that subscribers are located much denser in metropolitan regions; cf. Fig. 1 (a). In contrast, Fig. 1 (b) shows a second scenario where network devices of an agglomeration of different metropolitan regions overlap and thus are scattered with varying density.

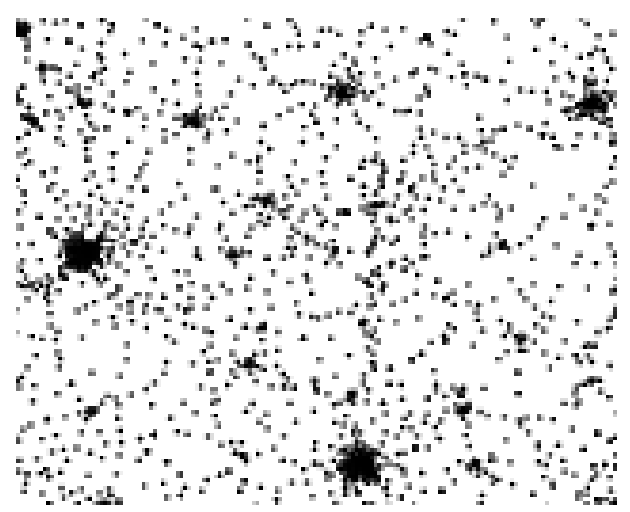

(a) Different metropolitan regions

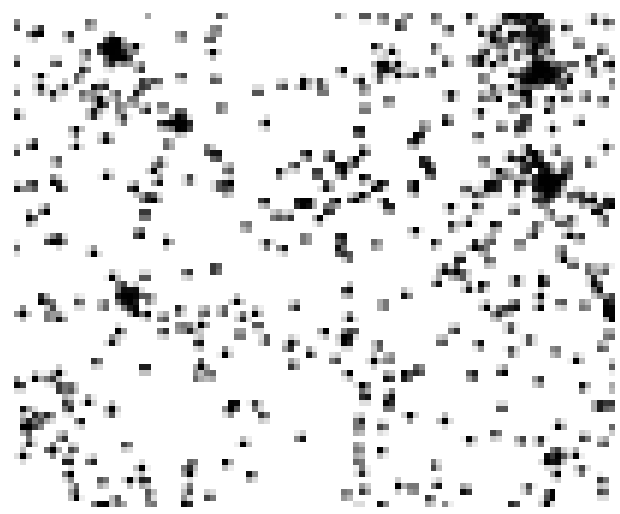

(b) Overlapping metropolitan regions

Fig. 1. Urban and rural locations of real network devices in a region of France

While the infrastructure along which some network devices are placed can be modelled by nationwide versions of the SSLM, there remains the crucial part to have at hand a flexible model for the geometry layer, i.e., a model that is able to appropriately display the spatial structure of the network geometry. Accurate estimations of the characteristics of the service zones associated to the network devices could then be easily obtained and used as components of cost or traffic models. Adopting classical stationary approaches is often too naive since spatial fluctu- 
ations are completely ignored. Truly instationary approaches like nonstationary Poissonian models with parametric intensity shapes can be used to obtain interesting results in the shape of integral formulations. But such approaches are limited and will quickly become complicated when closeness or overlapping of dense areas are to be taken into account. A possible way out of this dilemma is offered by a general framework including stationary cases that provide good approximations for instationary ones. Such a framework is, for example, given by modulated PoissonVoronoi tessellations (see Fig. 2) that, although being stationary models, are able to reflect instationary scenarios quite well. Hence, our proposed model can address real situations of nationwide networks as in Fig. 1 (a) or cases of overlapping dense areas like in Fig. 1 (b).

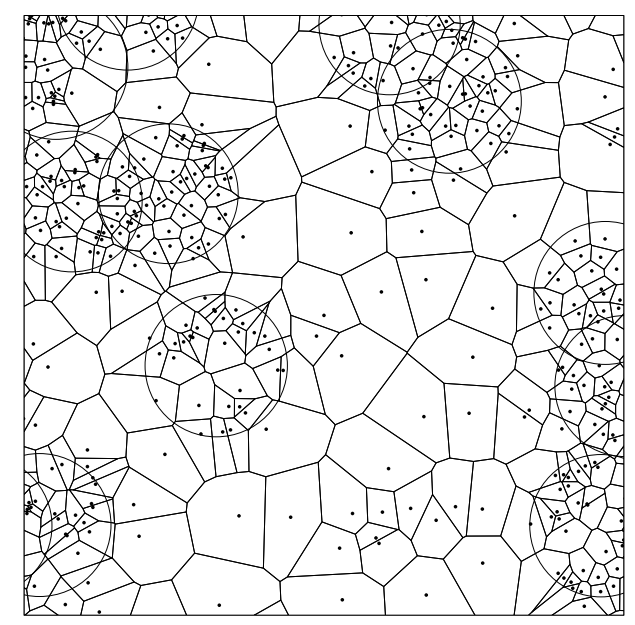

Fig. 2. Realization of a modulated Poisson-Voronoi tessellations

In this paper we analyze a special case of modulated Poisson-Voronoi tessellations where the modulation is generated by a Boolean germ-grain model with circular grains. Such a model can, for example, be used to model population densities or densities of network devices on nationwide scales. It is able to cover a wide variety of different scenarios due to the fact that the underlying point process is very flexible. The possible randomness of the radius of the grains allows to mimic the observed features of the towns lying in a given area and thus to achieve a proper analysis of a given region. Especially we are interested in the characteristics of socalled typical cells of these tessellations. In the stationary case, the typical cell can be regarded as a cell that is chosen at random out of the pool of all cells available. For ergodic tessellation models this means that we can study characteristics of the typical cell instead of averaging over very large sampling windows since the analysis of large sampling windows has some grave practical disadvantages like memory and runtime problems as well as problems occurring from edge-effects. Characteristics we are exploring in this context are area, perimeter and number of vertices of the typical cell, where one should notice that all these characteristics are random vari- 
ables in this setting. Apart from that, natural characteristics of interest are basic cost functionals like the mean distance from a randomly chosen location within the cell to its corresponding cell nucleus (centre). Such an easy-to-handle cost functional often serves as a surrogate for more sophisticated cost functionals used in practice like the subscriber line length. All these characteristics are useful tools in the cost analysis of telecommunication networks. In particular they serve as components for more sophisticated functionals which can be obtained by combining these basic functionals. Notice that often not only first moments (means) are of interest but also second or even higher moments in order to allow for an efficient risk analysis, for example. It is also important to notice that by looking at the functionals of the typical cell automatically functionals for the model on a global view are obtained due to ergodicity. This means that, e.g., by estimating the mean area of the typical cell, the mean area for the cells in a (very large) region is estimated.

After an introduction of the model of stationary modulated Poisson-Voronoi tessellations and the notion of its typical cell (Section 2), in Section 3 a simulation algorithm for the typical cell of modulated Poisson-Voronoi tessellations is given that is based on Palm calculus. Modifications for random radii of the circular grains of the underlying Boolean model are also discussed. In Section 4 the functional representing the mean distance from a random location to the corresponding cell nucleus is introduced and it is shown via usage of Neveu's exchange formula for Palm probability measures how to estimate the cost functional by simulating the typical cell of the modulated Poisson-Voronoi tessellation. Afterwards in Section 5 numerical examples are provided, dealing with some special cases like the Swiss cheese model introduced in Section 2.2.

Additionally, possibilities for statistical software testing of an implementation of the introduced algorithm are discussed here. Finally in Section 6 an outlook to possible extensions and to other modulated Poisson-type models is provided.

All implementations that have been done for the computation and the simulation of the typical cell of modulated Poisson-Voronoi tessellations and the corresponding cost functionals are integrated in the GeoStoch library. This JAVA-based library was developed by the Departments of Stochastics and Applied Information Processing of the University of Ulm in order to offer software tools designed for the analysis of spatial data with methods from stochastic geometry; see Mayer, Schmidt and Schweiggert [15] and http://www.geostoch.de/.

\section{Stationary modulated Poisson-Voronoi tessellations and their typi- cal cells}

This section introduces the mathematical model of a stationary modulated Poisson-Voronoi tessellation that is generated by a Boolean germ-grain model. For further details see appendices A.1-A.5. After the discussion of some special cases for such modulated Poisson-Voronoi tesssellations, a Palm representation for the corresponding typical cell is provided that is used in Section 3 to derive a simulation algorithm. 


\subsection{Stationary modulated Poisson-Voronoi tessellations}

In the following let $\Psi=\bigcup_{n \geq 1}\left(Y_{n}+M_{n}\right)$ be a Boolean germ-grain model (see (A.12)) where $\left\{Y_{n}\right\}$ is a stationary Poisson point process with intensity $\beta>0$ and where the $M_{n}$ are circular grains with a fixed radius $r$. In Fig. 3 realizations of $\left\{Y_{n}\right\}$ and $\Psi$ are displayed. Furthermore let $X=\left\{X_{n}\right\}_{n \geq 1}$ be a planar doubly-stochastic Poisson point process (also called Cox point process; see (A.4)) that has a random driving measure $\Lambda$ generated by $\Psi$ which is defined as

$$
\Lambda(d x)= \begin{cases}\lambda_{1} d x & \text { if } x \in \Psi, \\ \lambda_{2} d x & \text { if } x \notin \Psi,\end{cases}
$$

where $0 \leq \lambda_{1}, \lambda_{2}<\infty$ and $\max \left\{\lambda_{1}, \lambda_{2}\right\}>0$. Then the Voronoi tessellation $\tau_{X}$ induced by the Cox point process $X$ is called a modulated Poisson-Voronoi tessellation (see (A.14)). Analoguously, $X$ is referred to as a modulated Poisson process. We often call $\Psi$ the Boolean model corresponding to $X$ and $\tau_{X}$. In Fig. 2 a realization of a modulated Poisson-Voronoi tessellation is displayed.

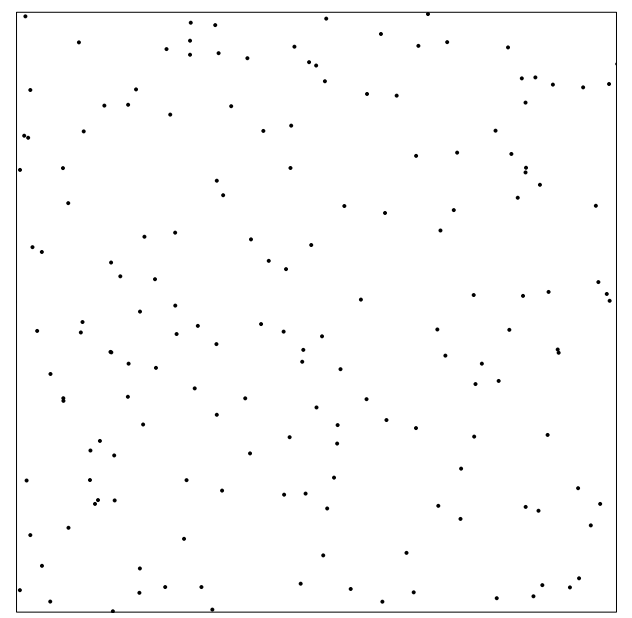

(a) Realization of a Poisson process

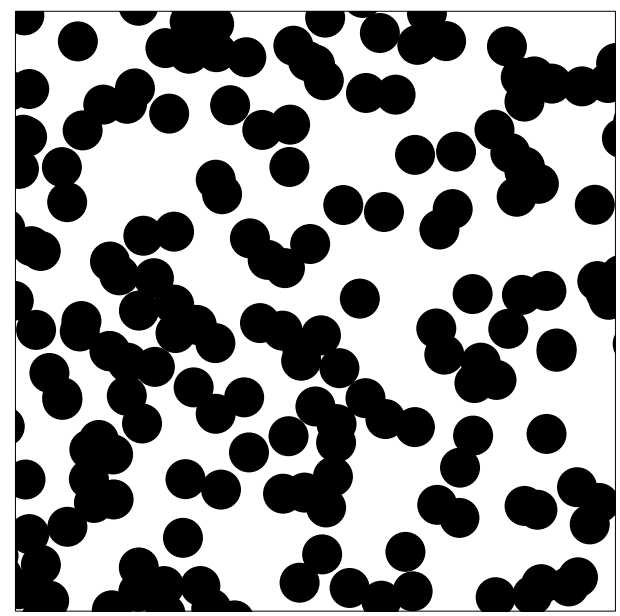

(b) Realization of a Boolean model

Fig. 3. Boolean germ-grain model

Due to the stationarity of $\Psi$ and the definition of $\Lambda$ given in (2.1), it is obvious that both $X$ and $\tau_{X}$ are stationary. Also, since the Boolean model $\Psi$ is an ergodic random closed set, both processes $X$ and $\tau_{X}$ are ergodic.

The intensity $\lambda$ of the modulated Poisson process $X$ can be computed as

$$
\lambda=\frac{\mathbb{E} X(B)}{|B|}=p \lambda_{1}+(1-p) \lambda_{2},
$$

where $p=\mathbb{P}(o \in \Psi)$ denotes the coverage probability of the (stationary) Boolean model $\Psi, X(B)=\#\left\{n: X_{n} \in B\right\}$, and $B \in \mathcal{B}\left(\mathbb{R}^{2}\right)$ is an arbitrary Borel set with positive and finite Lebesgue measure $|B|$. 


\subsection{Special cases of modulated Poisson-Voronoi tessellations}

The modulated Poisson-Voronoi tessellation introduced in Section 2.1 covers a wide variety of different models due to the fact that the underlying point process is very variable. The basic case is the ordinary stationary Poisson-Voronoi tessellation (PVT), where $\lambda_{1}=\lambda_{2}$ (Fig. 4 (a)). Notice that for this case the Poisson point process $X$ becomes independent from the Boolean model $\Psi$. Another interesting special case is the Swiss cheese model, where $\lambda_{1}=0$ (Fig. 4 (b)). This model might be of interest first of all as a limiting case. Secondly there exist cases when there are "forbidden zones," e.g., for antennas in densely populated regions. We will regard a numerical example for the Swiss cheese model type in Section 5.2 since interesting effects can be observed there. A further special case that is interesting for applications is the inner-city model, where $\lambda_{2}=0$ (Fig. 4 (c)). The underlying point process $X$ in such a case should not be confused with so-called Matern cluster processes since the intensity at a location covered by $\Psi$ is given as the constant value $\lambda_{1}$ no matter how many circles of $\Psi$ are covering a specific location.

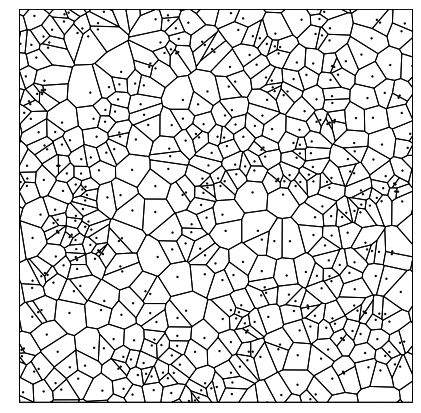

(a) Ordinary PVT

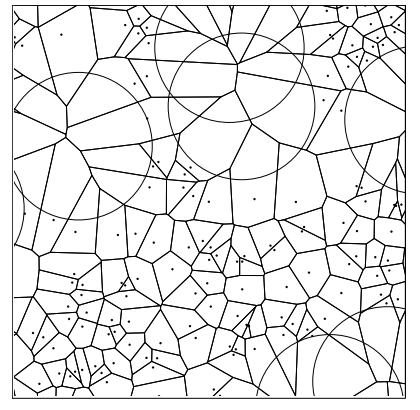

(b) Swiss cheese model

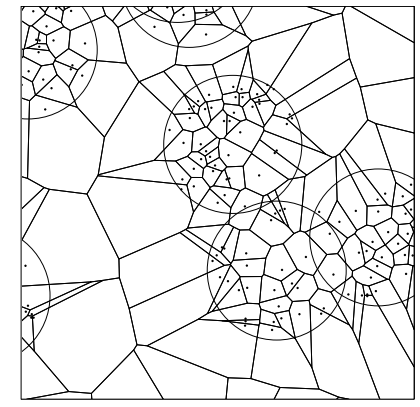

(c) Inner-city model

Fig. 4. Special cases of modulated Poisson-Voronoi tessellations

\subsection{Representation of the typical cell}

Using Palm calculus (see Sections A.3 and A.5), the typical cell of the modulated Poisson-Voronoi tessellation $\tau_{X}$ can be described as follows. Let the modulated Poisson process $X$ of nuclei have random driving measure $\Lambda$ given in (2.1) and let $\Psi$ be the corresponding Boolean model. Let $Q^{*}$ denote the Palm distribution of the stationary random measure $\Lambda$ (see for example Stoyan, Kendall and Mecke [18], p. 229) and let $X^{*}$ be a Cox point process with random driving measure $\Lambda^{*}$ having the distribution $Q^{*}$, where

$$
Q^{*}(\cdot)=\frac{\lambda_{1}}{\lambda} \mathbb{P}(\Lambda \in \cdot, o \in \Psi)+\frac{\lambda_{2}}{\lambda} \mathbb{P}(\Lambda \in \cdot, o \notin \Psi) .
$$

Then, under its Palm probability measure $\mathbb{P}_{X}^{*}$ (see (A.10)), the Cox point process $X$ has the same distribution as $\delta_{o}+X^{*}$ has under the original probability measure $\mathbb{P}$, i.e.,

$$
\mathbb{P}_{X}^{*}(X \in \cdot)=\mathbb{P}\left(\delta_{o}+X^{*} \in \cdot\right),
$$


where $\delta_{o}$ is the (deterministic) point process that consists solely of one single point at the origin $o$. Thus, the typical cell of $\tau_{X}$ has the same distribution as the Voronoi cell with nucleus at $o$ which is induced by the point process $\delta_{o}+X^{*}$. Notice that the Palm distribution $Q^{*}$ given in $(2.3)$ can be written as

$$
Q^{*}(\cdot)=p_{c} \mathbb{P}(\Lambda \in \cdot \mid o \in \Psi)+\left(1-p_{c}\right) \mathbb{P}(\Lambda \in \cdot \mid o \notin \Psi),
$$

where

$$
p_{c}=\frac{p \lambda_{1}}{\lambda}
$$

is the conditional coverage probability $p_{c}=\mathbb{P}_{X}^{*}(o \in \Psi)$ of the origin $o$ by the Boolean model $\Psi$ under the Palm probability measure $\mathbb{P}_{X}^{*}$, i.e., conditional to the event that $o$ belongs to the point process $X$, whereas $p=\mathbb{P}(o \in \Psi)$ represents the (unconditional) coverage probability of $o$ by $\Psi$ and $\lambda$ is the intensity of $X$. By (2.5) it becomes clear that the Cox point process $X^{*}$ is a mixture of two Cox point processes with random driving measure whose conditional distributions are given by $\mathbb{P}(\Lambda \in \cdot \mid o \in \Psi)$ and $\mathbb{P}(\Lambda \in \cdot \mid o \notin \Psi)$, respectively.

\section{Simulation algorithm}

In this section we introduce a simulation algorithm for the typical cell of modulated Poisson-Voronoi tessellations that are generated by the Boolean model with circular grains having fixed radius $r>0$. This algorithm is based on the Palm representation of the typical cell derived in Section 2.3. Finally, in Section 3.5, the case of random radii is also considered.

\subsection{Radial simulation of Poisson point processes}

The simulation algorithm used later on utilizes radial simulation of Poisson processes in $\mathbb{R}^{2}$, where radial in this context means that the simulated points have an increasing distance to the origin. For a more general description of radial generation of Poisson processes see Quine and Watson [16]. Recall that a point $x=\left(x_{1}, x_{2}\right) \in \mathbb{R}^{2}$ can be represented in polar coordinates as $x=(r, z)$, where $x_{1}=r \cos z$ and $x_{2}=r \sin z$. Consider a sequence of random variables $\left\{R_{i}\right\}_{i \geq 1}$ with $R_{0}<R_{1}<\cdots$ such that $\left\{R_{i}\right\}$ is a (linear) stationary Poisson point process in $(0, \infty)$ with parameter $\gamma$. Furthermore, consider another sequence $\left\{Z_{i}\right\}$ of independent and $U(0,2 \pi]$-distributed random variables, which is independent of $\left\{R_{i}\right\}$. Then the sequence $\left\{\left(\left(R_{i} / \pi\right)^{1 / 2}, Z_{i}\right)\right\}$ is a (two-dimensional) stationary Poisson point process in $\mathbb{R}^{2}$ with (planar) intensity $\gamma$.

In practice this means that a stationary Poisson point process in $\mathbb{R}^{2}$ can be generated radially by simulating independent random variables $T_{j} \sim \operatorname{Exp}(\gamma)$, and $V_{i} \sim U(0,2 \pi)$ and by putting

$$
R_{i}=\sum_{j=0}^{i} \log T_{j}, \quad i \geq 1
$$

and

$$
Z_{i}=V_{i}, \quad i \geq 1 .
$$




\section{F. Fleischer, C. Gloaguen, H. Schmidt, V. Schmidt and F. Schweiggert}

\subsection{Conditional simulation of modulated Poisson processes}

Equations (2.3)-(2.6) provide a theoretical basis for the simulation of the typical cell of the modulated Poisson-Voronoi tessellation $\tau_{X}$ that corresponds to the modulated Poisson process $X$. Recall that the typical cell of $\tau_{X}$ has the same distribution as the Voronoi cell with nucleus at $o$ which is induced by the point process $\delta_{o}+X^{*}$ given in (2.4). Hence the modulated Poisson process $X^{*}=\left\{X_{n}^{*}\right\}_{n \geq 1}$ has to be simulated, whose random driving measure has distribution $Q^{*}$ given in (2.5). Note that due to $(2.5)$ a simulation of $X^{*}$ requires a simulation of the Boolean model $\Psi^{*}$, conditional to the events that the origin is covered by $\Psi$ or not. In other words $\Psi^{*}$ has to be simulated conditional to the event that $o \in X$. The simulation of $X^{*}$ and $\Psi^{*}$ is performed radially, i.e., with increasing distance to the origin, and in an alternating fashion between the points of $X^{*}$ and the germs of $\Psi^{*}$. As an initial step a point $X_{0}^{*}$ is placed in the origin (Fig. 5 (a)), thereby representing the (degenerate) point process $\delta_{o}$ in (2.4). Then, it is determined by a Bernoulli experiment with success probability $p_{c}$ given in (2.6) if $X_{0}^{*}=o$ is covered by $\Psi$ or not. If $o \in \Psi$ then the distance of $Y_{1}^{*}$, the germ of $\Psi^{*}$ which is nearest to $X_{0}^{*}$, to the origin has to be less than or equal to $r$, otherwise, i.e., if $o \notin \Psi$ it has to be bigger than $r$. Therefore the distance of the first germ $Y_{1}^{*}$ to the origin has to be simulated conditional to $o \in \Psi$ or $o \notin \Psi$, respectively (Fig. 5 (b)). In practice this means that a proposal distance $R_{1}$ of the first germ $Y_{1}^{*}$ to the origin is generated according to (3.1) with $\gamma=\beta$, where $\beta$ represents the intensity of the germs as defined in Section 2.1. This proposal distance is accepted or rejected based on $R_{1} \leq r$ or $R_{1}>r$, respectively. In case of a rejection another proposal distance $R_{1}$ of $Y_{1}^{*}$ to the origin is generated. Afterwards further points $X_{i}=\left(R_{i}, Z_{i}\right)$ are simulated radially according to (3.1) and (3.2) with intensity $\gamma=\max \left\{\lambda_{1}, \lambda_{2}\right\}$. For each such point it is checked whether it is covered by $\Psi^{*}$ or not (Fig. 5 (c)). This check is performed by simulating further germs $Y_{j}^{*}$ of $\Psi^{*}$ until either the distance of a germ to $X_{i}$ becomes smaller than or equal to $r$ or if the distance of $Y_{j}^{*}$ to the origin becomes greater than $\left|X_{i}\right|+r$, where $\left|X_{i}\right|$ denotes the distance of $X_{i}$ to the origin. In the first case $X_{i}$ is covered by $\Psi^{*}$, in the second, it is clear that it is not covered. Notice that it is important to retain all germs simulated for further checks, e.g., of the point $X_{i+1}$. After we have checked whether $X_{i}$ is covered by the conditional Boolean model $\Psi^{*}$ or not, in one of the two cases a thinning procedure has to be performed. So, if without loss of generality $\lambda_{1}<\lambda_{2}$ and $X_{i} \in \Psi^{*}$ then the probability of discarding $X_{i}$ is given by $1-\lambda_{1} / \lambda_{2}$. Once more it is important to retain the distance of $X_{i}$ to the origin as a starting value for the simulation of $X_{i+1}$, even if $X_{i}$ is discarded in the thinning procedure in order to obtain correct results. Altogether, this method leads to a simulation of $X^{*}=\left\{X_{n}^{*}\right\}_{n \geq 1}$ by an alternating simulation of a stationary Poisson point process $X_{\max }$ with intensity $\gamma=\max \left\{\lambda_{1}, \lambda_{2}\right\}$ and a conditional Boolean model $\Psi^{*}$ and by applying the thinning procedure described above (Fig. 5 (d)). Notice that unconditional simulation of a (stationary) modulated Poisson process in the plane can be performed in a similar way by an alternating radial simulation of $X_{\max }$ and the (unconditional) Boolean model $\Psi$. 


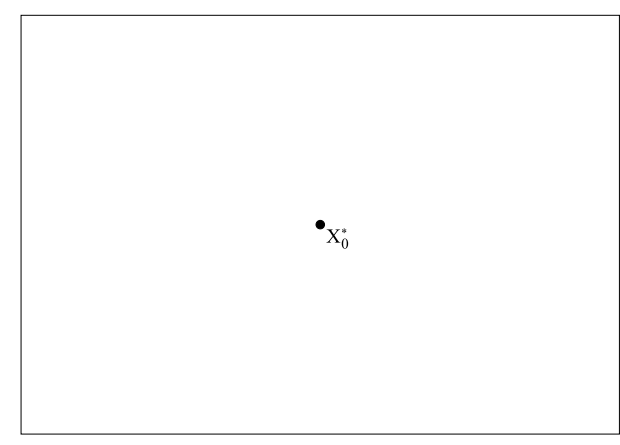

(a) Point $X_{0}^{*}$ at the origin

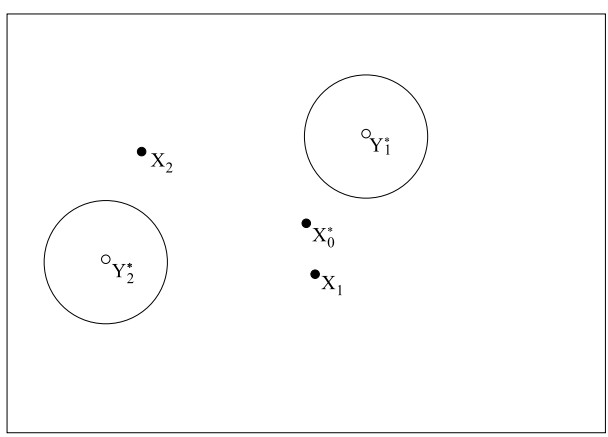

(c) For $X_{1}$ all necessary information about $\Psi^{*}$ is given since $\left|Y_{2}^{*}\right|>\left|X_{1}\right|+r$. For $X_{2}$ more information about $\Psi^{*}$ is needed.

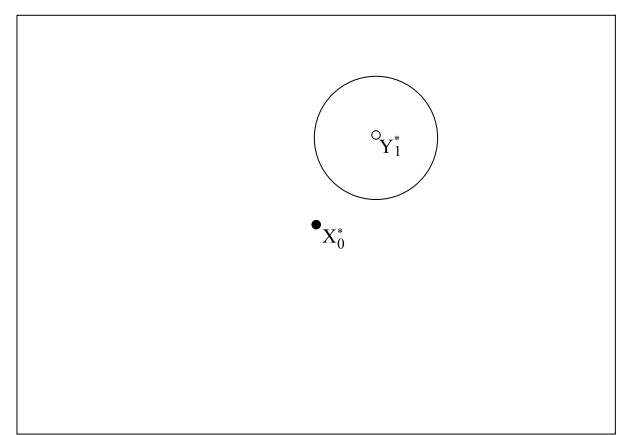

(b) First grain with midpoint $Y_{1}^{*}$

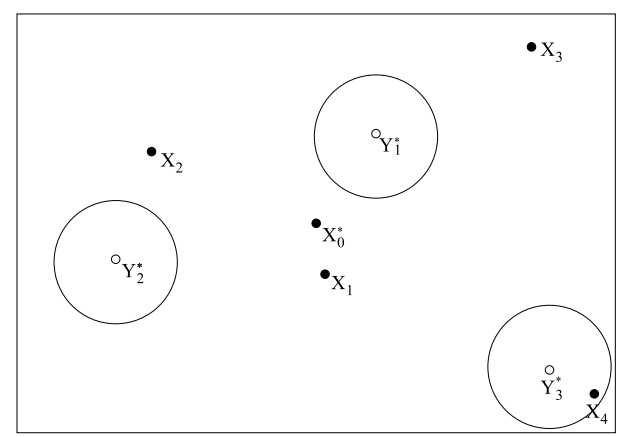

(d) Further alternating simulation of $X^{*}$ and $\Psi^{*}$

Fig. 5. Algorithm, initial steps and alternating simulation

\subsection{Construction of initial cell}

Based on the radial simulation of the modulated Poisson process $X^{*}$ as it was explained in Section 3.2 an initial cell for the typical cell is constructed next. This means that if for each point $X_{n}^{*}$ of $X^{*}=\left\{X_{n}^{*}\right\}_{n \geq 1}$ the perpendicular bisector $\left(X_{0}^{*}, X_{n}^{*}\right)$ is regarded we are interested in a minimal integer $n \geq 3$ such that $X_{0}^{*}$ is for the first time surrounded by a convex polygon formed by these bisectors. In Fig. 6 a procedure for the construction of such an initial cell is visualized (see also Quine and Watson [16] and Wendel [20]). The lines $\overline{X_{1}^{*} X_{0}^{*}}$ and $\overline{X_{2}^{*} X_{0}^{*}}$ form a cone $S_{2}$ with respect to the opposite side of $X_{0}^{*}$. If the (next nearest) point $X_{3}^{*}$ lies inside of this cone the algorithm stops and an initial cell can be constructed using the bisectors $\left(X_{0}^{*}, X_{1}^{*}\right),\left(X_{0}^{*}, X_{2}^{*}\right)$ and $\left(X_{0}^{*}, X_{3}^{*}\right)$. Otherwise the cone $S_{3}$ is taken as the maximal cone formed by two of the three lines $\overline{X_{1}^{*} X_{0}^{*}}, \overline{X_{2}^{*} X_{0}^{*}}$ and $\overline{X_{3}^{*} X_{0}^{*}}$ on the opposite side of $X_{0}^{*}$. Afterwards the point $X_{4}^{*}$ is taken into account with respect to $S_{3}$ (Fig. 6 (a)). This procedure is repeated until $X_{i+1}^{*} \in S_{i}$. With probability 1 this algorithm stops after a finite number of steps (see Wendel [20] for the case of stationary Poisson processes) and an initial cell can be constructed by using the corresponding bisectors (Fig. 6 (b)). 


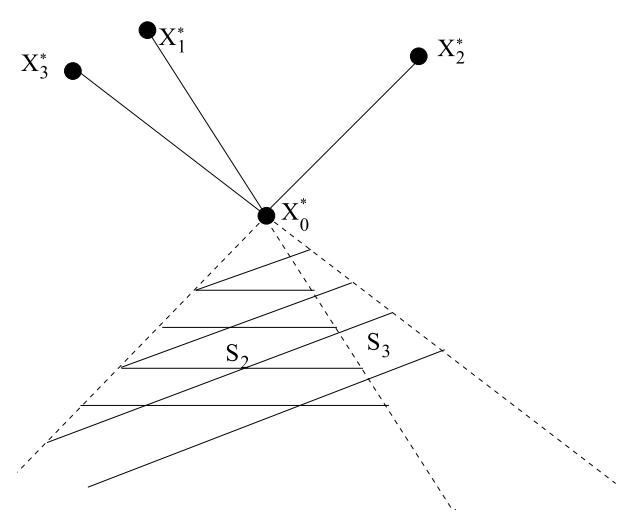

(a) Stopping criterion for initial cell

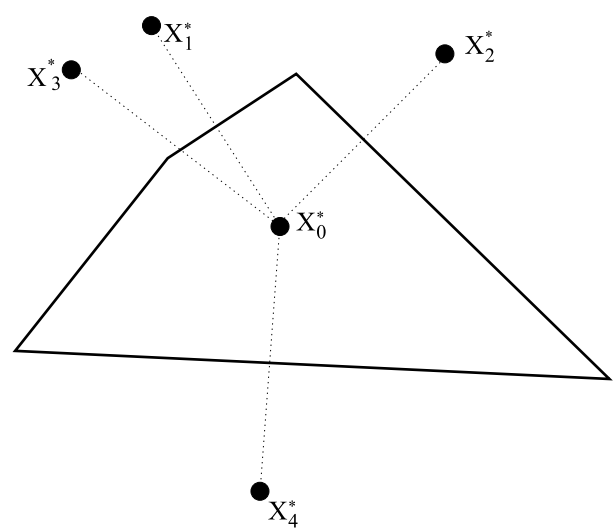

(b) Construction of initial cell using bisectors

Fig. 6. Stopping criterion for initial cell and its construction

\subsection{Simulation of the typical cell}

After the creation of an initial cell a stopping criterion for the simulation of the typical cell can be provided (see Quine and Watson [16]). If $d_{\max }$ denotes the maximal distance of the vertices of the initial cell to the the origin $o\left(=X_{0}^{*}\right)$ then the simulation of the points $X_{i}^{*} \in X^{*}$ has to be continued until the distance of $X_{i}^{*}$ to $o$ is bigger than $2 d_{\max }$ (Fig. 7 ). Notice that $d_{\max }$ might be reduced during alterations of the cell (Fig. 7 (b) and Fig. 7 (c)) and therefore the stopping criterion has to be adapted accordingly in order to ensure faster runtimes. The final result after fulfilling the stopping criterion is a realization of the typical cell. (Fig. 7 (d)).

\subsection{Modifications for random radii}

In order to simulate the typical cell for modulated Poisson-Voronoi tessellations in the case where the radius $R$ of the circles of the corresponding Boolean model $\Psi$ is random but bounded (e.g., $R \sim U[r-\delta, r+\delta]$ with $0<\delta<r$ ) two modifications to the algorithm introduced above for deterministic radius $r$ have to be applied. It is important to notice that, with respect to the simulation of the modulated Poisson process $X^{*}$, in the case that the origin is covered by the conditional Boolean model $\Psi^{*}$, the grain generated by the first germ $Y_{1}^{*}$ of $\Psi^{*}$ with random radius $R_{1}^{*}$ does not necessarily cover the origin $o$. However it is possible that another grain covers $o$. Therefore, after determining whether $X_{0}^{*}=o$ is covered, the conditional radial simulation of the distances of the germs of $\Psi^{*}$ to the origin together with the radii of the corresponding grains has to be performed in a way such that in the case $o \in \Psi^{*}$ at least one grain $Y_{i}^{*}+M_{i}^{*}$ covers $X_{0}^{*}$. On the other hand, if $o \notin \Psi^{*}$ one has to simulate grains $Y_{i}^{*}+M_{i}^{*}$ that do not cover $X_{0}^{*}$ until the distance of their corresponding germs to $X_{0}^{*}$ is bigger than the maximal possible radius $r_{\max }$ (in the example of uniform distribution above $r_{\max }=r+\delta$ ). In practice this means that given $o \in \Psi^{*}$ or $o \notin \Psi^{*}$ a proposal sequence of germs $\left\{Y_{i}+M_{i}\right\}$ is radially generated for $i=0, \ldots, I_{\max }$, where $\left|Y_{I_{\max }}\right|>r_{\max }$. Afterwards it is 


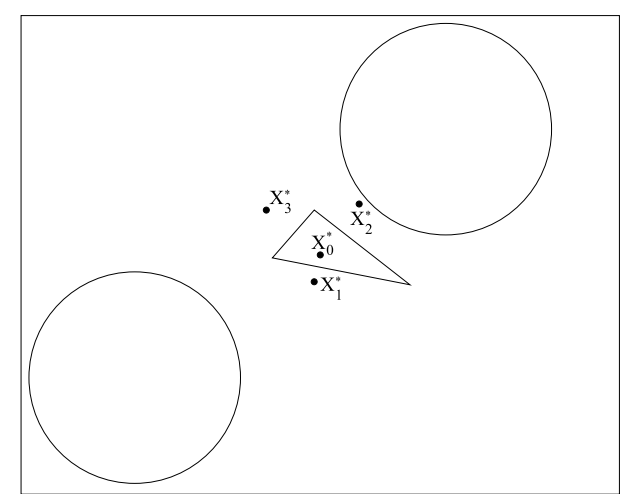

(a) Initial cell

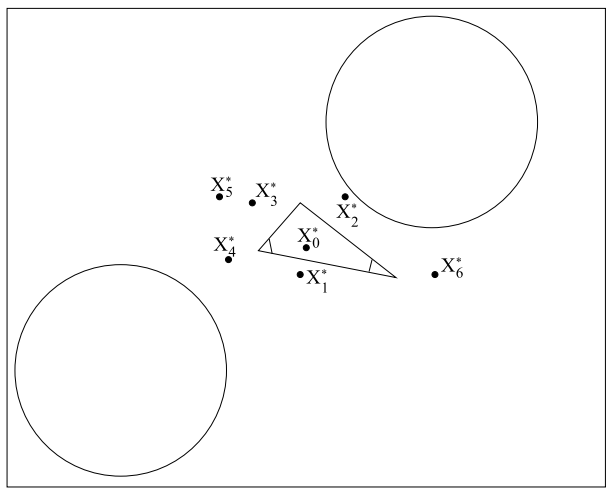

(c) Further alteration of initial cell by $X_{6}^{*}$

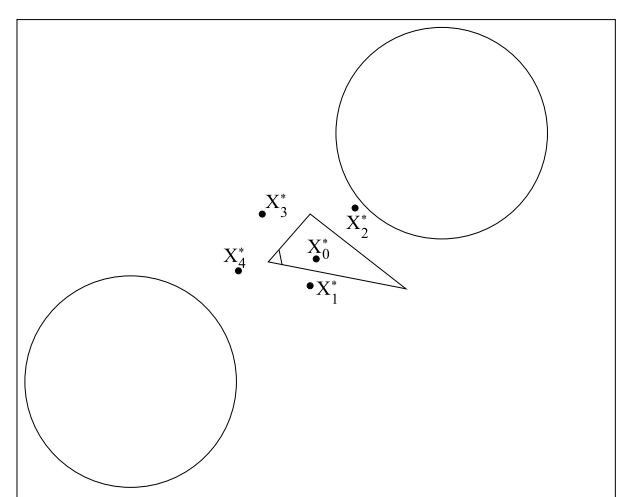

(b) First alteration of initial cell by $X_{4}^{*}$

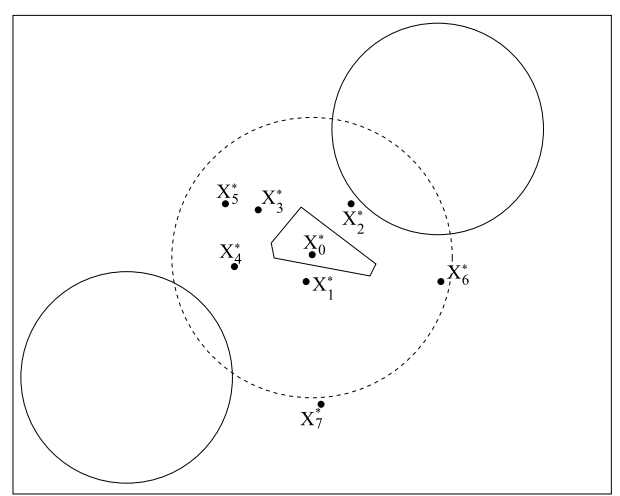

(d) Realization of the typical cell

Fig. 7. Alterations of initial cell and final realization of the typical cell

checked whether this sequence fulfills the given condition or not. In the first case the sequence is accepted and the simulation of the grains is continued radially with the grain $Y_{I_{\max }+1}+M_{I_{\max }+1}$, otherwise a new proposal sequence is radially generated by starting at the origin again. This procedure is repeated until a sequence is found that can be accepted. An analogous modification has to be performed with respect to the necessary amount of grains that have to be simulated in order to know if a point $X_{i}$ is covered by $\Psi^{*}$ or not. In the case of a deterministic radius $r$ of the grains it is sufficient to simulate until the distance of the germs of $\Psi^{*}$ to the origin is bigger than $\left|X_{i}\right|+r$. Now for random radii, the necessary distance to the origin has to be bigger than $\left|X_{i}\right|+r_{\max }$, where again $r_{\max }$ is the maximal possible radius $(r+\delta$ in the example).

\section{Functionals built on the typical cell}

As a basic example of functionals that can be built on the typical cell, we consider here the average distance from a randomly chosen location to the nearest nucleus of the modulated Poisson-Voronoi tessellation. The choice of this location 
might take place purely random, in other words following the distribution of a stationary Poisson point process, or again might be taken from a modulated Poisson process connected to the same Boolean model that the modulated Poisson process of nuclei uses.

\subsection{Definition via Palm probability measure}

Suppose that $\lambda_{1}, \lambda_{2}, \tilde{\lambda}_{1}, \tilde{\lambda}_{2} \geq 0$ are non-negative numbers such that $\max \left\{\lambda_{1}, \lambda_{2}\right\}>0$ and $\max \left\{\tilde{\lambda}_{1}, \tilde{\lambda}_{2}\right\}>0$. Let $X=\left\{X_{n}\right\}_{n \geq 1}$ be a modulated Poisson process connected to a Boolean model $\Psi$ via the random driving measure

$$
\Lambda(d x)= \begin{cases}\lambda_{1} d x & \text { if } x \in \Psi \\ \lambda_{2} d x & \text { if } x \notin \Psi\end{cases}
$$

and let $\left\{\tilde{X}_{n}\right\}_{n \geq 1}$ be another modulated Poisson process connected to the same Boolean model $\Psi$ via the random driving measure

$$
\tilde{\Lambda}(d x)= \begin{cases}\tilde{\lambda}_{1} d x & \text { if } x \in \Psi \\ \tilde{\lambda}_{2} d x & \text { if } x \notin \Psi\end{cases}
$$

Assume that the modulated Poisson processes $\left\{X_{n}\right\}$ and $\left\{\tilde{X}_{n}\right\}$ are conditionally independent, given $\Psi$. Furthermore, if $N\left(\tilde{X}_{n}\right)$ denotes the location of the nearest (in the Euclidean sense) point of $X$ with respect to $\tilde{X}_{n}$ consider the marked point process $\tilde{X}=\left\{\tilde{X}_{n},\left|\tilde{X}_{n}-N\left(\tilde{X}_{n}\right)\right|\right\}_{n \geq 1}$, where $|\cdot|$ denotes the Euclidean norm. The intensities of $X$ and $\tilde{X}$ are given by $\lambda=p \lambda_{1}+(1-p) \lambda_{2}$ and $\tilde{\lambda}=p \tilde{\lambda}_{1}+(1-p) \tilde{\lambda}_{2}$, respectively, where $p=\mathbb{P}(o \in \Psi)$. The functional we are especially interested in is the average distance $c$ from the typical point of $\tilde{X}$ to its nearest point of $X$. Using the Palm probability measure $\mathbb{P}_{\tilde{X}}^{*}$ for $\tilde{X}$ (see (A.10)) we can express $c$ as

$$
c=\mathbb{E}_{\tilde{X}}|N(o)|
$$

where $\mathbb{E}_{\tilde{X}}$ denotes expectation with respect to $\mathbb{P}_{\tilde{X}}^{*}$. Notice that due to the ergodicity of $\tilde{X}$ it is possible to express the expectation $c$ as the limit of spatial averages with respect to an averaging sequence $\left\{W_{i}\right\}_{i \geq 1}$ of unboundedly increasing sampling windows $W_{i}$. This means that with probability 1 (see for example Daley and Vere-Jones [9])

$$
c=\lim _{i \rightarrow \infty} \frac{1}{\#\left\{n: X_{n} \in W_{i}\right\}} \sum_{n \geq 1} \mathbb{1}_{W_{i}}\left(\tilde{X}_{n}\right)\left|\tilde{X}_{n}-N\left(\tilde{X}_{n}\right)\right| .
$$

\subsection{Application of Neveu's formula}

The following theorem allows for a practically more feasible representation of the functional $c=\mathbb{E}_{\tilde{X}}|N(o)|$ given in (4.3). Thereby a more efficient way of computing an approximation for $c$ is derived. 
Theorem 4.1. Consider the modulated Poisson process $X=\left\{X_{n}\right\}_{n \geq 1}$ and the (marked) modulated Poisson process $\tilde{X}=\left\{\tilde{X}_{n},\left|\tilde{X}_{n}-N\left(\tilde{X}_{n}\right)\right|\right\}_{n \geq 1}$ whose (random) driving measures $\Lambda$ and $\tilde{\Lambda}$ are generated by the same Boolean model $\Psi$ according to (4.1) and (4.2), respectively. Then,

$$
\mathbb{E}_{\tilde{X}}|N(o)|=\frac{\lambda}{\tilde{\lambda}} \mathbb{E}_{X}\left(\tilde{\lambda}_{1} \int_{\Xi_{X} \cap \Psi}|u| d u+\tilde{\lambda}_{2} \int_{\Xi_{X} \cap \Psi^{c}}|u| d u\right),
$$

where $\Xi_{X}$ denotes the Voronoi cell induced by $X$, which contains the origin, and $\mathbb{E}_{X}$ is the expectation taken with respect to $\mathbb{P}_{X}^{*}$.

Proof. The proof of Theorem 4.1 is based on Neveu's exchange formula (see (A.11)) for jointly stationary point processes, which are defined on a common probability space $(\Omega, \mathcal{A}, \mathbb{P})$ equipped with some flow $\left\{\theta_{x}: x \in \mathbb{R}^{2}\right\}$. We use (A.11) with $X_{D}$ and $\tilde{X}_{\tilde{D}}$ being equal to $X$ and $\tilde{X}$, respectively. Thus, the mark space $D$ will be omitted and $\tilde{D}=[0, \infty)$. Consider the function $f: \mathbb{R}^{2} \times[0, \infty) \times \Omega \rightarrow[0, \infty)$ given by

$$
f(x, \tilde{g}, \omega)= \begin{cases}\tilde{g} & \text { if } X\left(\theta_{-x} \omega, B_{|x|}^{\neq}(x)\right)=0, \\ 0 & \text { otherwise }\end{cases}
$$

for any $x \in \mathbb{R}^{2}, \tilde{g} \geq 0$, and $\omega \in \Omega$, where $B_{|x|}^{\neq}(x)=\left\{y \in \mathbb{R}^{2}:|y-x|<|x|\right\}$. Notice that if $x \in \mathbb{R}^{2}$ is an atom of the counting measure $X(\omega, \cdot)$, then $f(-x, \tilde{g}, \omega)=\tilde{g}$ only if there are no other atoms of $X(\omega, \cdot)$ which have a distance of less than $|x|$ to the origin. Thus, applying Neveu's exchange formula (Theorem A.1), we obtain that

$$
\begin{aligned}
\mathbb{E}_{\tilde{X}}|N(o)| & =\int_{\Omega \times \tilde{D}} \int_{\mathbb{R}^{2}} f(-x, \tilde{g}, \omega) X(\omega, d x) \mathbb{P}_{\tilde{X}}(d(\omega, \tilde{g})) \\
& =\frac{\lambda}{\tilde{\lambda}} \int_{\Omega} \int_{\mathbb{R}^{2} \times \tilde{D}} f\left(x, \tilde{g}, \theta_{x} \omega\right) \tilde{X}(\omega, d(x, \tilde{g})) \mathbb{P}_{X}(d \omega) .
\end{aligned}
$$

Given the Boolean model $\Psi$ the inner integral on the right hand side of (4.7) can be expressed as

$$
\begin{aligned}
\int_{\mathbb{R}^{2} \times \tilde{D}} f\left(x, \tilde{g}, \theta_{x} \omega\right) \tilde{X}(\omega, d(x, \tilde{g}))= & \int_{\left(\mathbb{R}^{2} \cap \Psi\right) \times \tilde{D}} f\left(x, \tilde{g}, \theta_{x} \omega\right) \tilde{X}(\omega, d(x, \tilde{g})) \\
& +\int_{\left(\mathbb{R}^{2} \cap \Psi^{c}\right) \times \tilde{D}} f\left(x, \tilde{g}, \theta_{x} \omega\right) \tilde{X}(\omega, d(x, \tilde{g})) .
\end{aligned}
$$

Furthermore, given $\Psi$ and the Voronoi cell $\Xi_{X}$ of $X$ that contains the origin, the random number of points of $\tilde{X}$ in $\Xi_{X} \cap \Psi$ is Poisson distributed with expectation $\eta_{1}=\tilde{\lambda}_{1}\left|\Xi_{X} \cap \Psi\right|$, while the random number of points of $\tilde{X}$ in $\Xi_{X} \cap \Psi^{c}$ is Poisson distributed with expectation $\eta_{2}=\tilde{\lambda}_{2}\left|\Xi_{X} \cap \Psi^{c}\right|$. Thus, by the definition of the 
function $f$ given in (4.6), the first integral on the right side of (4.8) can be written in the form

$$
\begin{aligned}
& \int_{\left(\mathbb{R}^{2} \cap \Psi\right) \times \tilde{D}} f\left(x, \tilde{g}, \theta_{x} \omega\right) \tilde{X}(\omega, d(x, \tilde{g})) \\
& =\int_{\left(\Xi_{X} \cap \Psi\right) \times \tilde{D}} f\left(x, \tilde{g}, \theta_{x} \omega\right) \tilde{X}(\omega, d(x, \tilde{g})) \\
& =\sum_{k=1}^{\infty} e^{-\eta_{1}} \frac{\eta_{1}^{k}}{k !} \int_{\Xi_{X} \cap \Psi} \cdots \int_{\Xi_{X} \cap \Psi} \sum_{i=1}^{k} \frac{\left|u_{i}\right|}{\left|\Xi_{X} \cap \Psi\right|^{k}} d u_{1} \cdots d u_{k},
\end{aligned}
$$

due to the conditional independence and uniform distribution of the points of $\tilde{X}$ in $\Xi_{X} \cap \Psi$. Hence,

$$
\begin{aligned}
\int_{\left(\mathbb{R}^{2} \cap \Psi\right) \times \tilde{D}} f\left(x, \tilde{g}, \theta_{x} \omega\right) \tilde{X}(\omega, d(x, \tilde{g})) & =\sum_{k=1}^{\infty} e^{-\eta_{1}} \frac{\eta_{1}^{k}}{k !} \frac{k}{\left|\Xi_{X} \cap \Psi\right|} \int_{\Xi_{X} \cap \Psi}|u| d u \\
& =\tilde{\lambda}_{1} \int_{\Xi_{X} \cap \Psi}|u| d u
\end{aligned}
$$

Analogously, it can be shown that

$$
\begin{aligned}
\int_{\left(\mathbb{R}^{2} \cap \Psi^{c}\right) \times \tilde{D}} f\left(x, \tilde{g}, \theta_{x} \omega\right) \tilde{X}(\omega, d(x, \tilde{g})) & =\sum_{k=1}^{\infty} e^{-\eta_{2}} \frac{\eta_{2}^{k}}{k !} \frac{k}{\left|\Xi_{X} \cap \Psi^{c}\right|} \int_{\Xi_{X} \cap \Psi^{c}}|u| d u \\
& =\tilde{\lambda}_{2} \int_{\Xi_{X} \cap \Psi^{c}}|u| d u .
\end{aligned}
$$

Altogether we get that

$$
\mathbb{E}_{\tilde{X}}|N(o)|=\frac{\lambda}{\tilde{\lambda}} \mathbb{E}_{X}\left(\tilde{\lambda}_{1} \int_{\Xi_{X} \cap \Psi}|u| d u+\tilde{\lambda}_{2} \int_{\Xi_{X} \cap \Psi^{c}}|u| d u\right),
$$

which completes the proof of the theorem.

In the special case that $\tilde{\lambda}_{1}=\tilde{\lambda}_{2}$, i.e., $\left\{\tilde{X}_{n}\right\}$ is an ordinary Poisson process (in the sense of (A.3)) Theorem 4.1 can be restated as follows.

Corollary 4.2. Suppose that $\tilde{\lambda}_{1}=\tilde{\lambda}_{2}$, i.e., $\left\{\tilde{X}_{n}\right\}$ is a stationary Poisson point process with intensity $\tilde{\lambda}$. Then

$$
\mathbb{E}_{\tilde{X}}|N(o)|=\lambda \mathbb{E}_{X} \int_{\Xi_{X}}|u| d u .
$$

Note that (4.9) shows in particular that $\mathbb{E}_{\tilde{X}}|N(o)|$ does not depend on $\tilde{\lambda}$. In the Poisson case, i.e., if $\lambda_{1}=\lambda_{2}$ and $\tilde{\lambda}_{1}=\tilde{\lambda}_{2}$, the cost functional $c=\mathbb{E}_{\tilde{X}}|N(o)|$ can be analytically computed as (see also Bacelli, Klein, Lebourges and Zuyev [4] and Błaszczyszyn and Schott [7])

$$
\mathbb{E}_{\tilde{X}}|N(o)|=\lambda \mathbb{E}_{X} \int_{\Xi_{X}}|u| d u=\lambda \int_{\mathbb{R}^{2}}|u| \exp \left(-\lambda \pi|u|^{2}\right) d u=\frac{1}{2 \sqrt{\lambda}} .
$$




\subsection{Estimation by Monte Carlo simulation}

Theorem 4.1 provides a useful approach for the construction of an estimator for the cost functional $c=\mathbb{E}_{\tilde{X}}|N(o)|$ which is based on Monte Carlo simulation. Let $\left(\Xi_{1}^{*}, \Psi_{1}^{*}\right), \ldots,\left(\Xi_{n}^{*}, \Psi_{n}^{*}\right)$ be a sequence of independent copies of $\left(\Xi_{X}, \Psi\right)$ under the Palm probability measure $P_{X}^{*}$. Then an unbiased and consistent estimator for $c$ is given by

$$
\hat{c}=\frac{\lambda}{\tilde{\lambda}} \frac{1}{n} \sum_{i=1}^{n} \int_{\Xi_{i}^{*}}|u| \tilde{\Lambda}_{i}(d u),
$$

where

$$
\tilde{\Lambda}_{i}(d u)= \begin{cases}\tilde{\lambda}_{1} d u & \text { if } x \in \Psi_{i}^{*}, \\ \tilde{\lambda}_{2} d u & \text { if } x \notin \Psi_{i}^{*} .\end{cases}
$$

The estimator $\hat{c}$ will be used in Section 5.2 in order to obtain numerical results for some example scenarios. Notice that if $\tilde{\lambda}_{1}=\tilde{\lambda}_{2}$ then the integral $\int_{\Xi_{i}^{*}}|u| \tilde{\Lambda}_{i}(d u)$ appearing in (4.11) is computed analytically, otherwise it is computed via numerical approximation. This is due to the fact that in the first case, by applying (4.9), we are able to rewrite the integral as an integral with respect to the Lebesgue measure. If $\tilde{\lambda}_{1} \neq \tilde{\lambda}_{2}$ integration must be performed with respect to the measure $\tilde{\Lambda}_{i}$ and therefore the shape of $\Psi_{i}^{*}$ has to be taken into account. This makes an analytical solution of the integral difficult to achieve.

Another important fact concerning a numerical evaluation is that it is not necessary to simulate any points of $\tilde{X}$ in order to apply the estimator given in (4.11).

\section{Implementation and numerical examples}

In this section we look at possible testing approaches for implementations of the algorithm introduced in Section 3. In a second part numerical results for different scenarios are regarded.

\subsection{Tests of implemented algorithm}

In our context, apart from traditional testing methods for software tests, in particular methods for testing of software with random output are of interest. In the following we want to mention some examples for such tests, where a more detailed discussion of random software testing can, for example, be found in Gloaguen, Fleischer, Schmidt and Schmidt [11] and Mayer and Guderlei [14]. Basically two different testing methods have been applied to the implemented algorithm.

A first testing method was constructed by using known theoretical formulae like the equality between the intensity $\lambda_{\tau}$ of the tessellation model $\tau_{X}$ and the reciprocal of the mean area $\mathbb{E}\left(\left|\Xi^{*}\right|\right)$ of the corresponding typical cell; see (A.13). In particular we tested if the algorithm provides correct estimates for the mean area $1 / \lambda_{\tau}$ of $\Xi^{*}$. This was achieved by constructing an asymptotically Gaussian distributed test statistic. Another example for a known theoretical formula that we used to construct tests for our software was the cost functional in the case of ordinary stationary Poisson processes; see (4.10). 
A different testing technique is to obtain tests by utilizing certain scaling properties of the underlying tessellation model, meaning that for different model parameter sets there is the same underlying random structure, only on a different length scale. In our specific model we get that for the three parameters $c_{1}=p, c_{2}=\lambda_{1} / \beta$ and $c_{3}=\lambda_{2} / \beta$ such a scaling invariance can be realized. Hence if for two models with parameter vectors $\left(p^{(1)}, \beta^{(1)}, \lambda_{1}^{(1)}, \lambda_{2}^{(1)}\right)$ and $\left(p^{(2)}, \beta^{(2)}, \lambda_{1}^{(2)}, \lambda_{2}^{(2)}\right)$ the relationship $\left(c_{1}^{(1)}, c_{2}^{(1)}, c_{3}^{(1)}\right)=\left(c_{1}^{(2)}, c_{2}^{(2)}, c_{3}^{(2)}\right)$ holds then they represent the same random structure but on a different scale. This fact can be used to construct tests similar to the tests for known theoretical formulae. For example, in such a case we have that $\beta^{(1)} \mathbb{E}\left|\Xi^{*(1)}\right|=\beta^{(2)} \mathbb{E}\left|\Xi^{*(2)}\right|$ which can be used as a theoretical formula in order to develop an asymptotic Gaussian test.

With consideration to the test results we obtained for our implementations it suffices here to say that they showed the expected behavior. Hence we may assume that the implemented algorithms for the simulation of the typical cell and the computation of the cost functional work correctly.

\subsection{Some numerical results}

With regard to numerical evaluations of the modulated Poisson-Voronoi tessellations considered in this paper it can be stated that due to the relatively large number of parameters involved, a complete analysis is almost impossible to achieve. Therefore we only concentrate on a specific scenario to show some of the interesting effects that appear. The scenario we want to consider consists of few large grains, where $p=0.6$ and $\beta=0.2$ that leads to a fixed radius $r=1.20761$. We assume a fixed total intensity $\lambda=12$ such that the mean area of the typical Voronoi cell remains constant as $\mathbb{E}\left|\Xi^{*}\right|=\lambda^{-1}=0.8333$. Such a scenario is realized in Fig. 4 (b) for $\lambda_{1}=0$.

As a first example we let the parameter $\lambda_{1}$ tend to 0 and regard the behavior of the distribution for the perimeter of the typical cell (Fig. 8). In other words we observe the behavior with regard to a transition to the Swiss cheese model. Notice that for each pair $\left(\lambda_{1}, \lambda_{2}\right)$ the sample size is given as $n=2000000$, while each bar of the histogram has a width of 0.05 . For the case of an ordinary stationary Poisson-Voronoi tessellation (Fig. 8 (a)) a symmetrical look of the histogram for the perimeter of the typical cell can be observed. This changes as $\lambda_{1}$ tends to 0 , resulting in a shape that is skewed to the left. Another interesting effect that can be observed is the existence of a second local maximum for the histogram, especially for very small values of $\lambda_{1}$. This is mainly caused by the cells that cover the grains of the corresponding Boolean model since inside of the Boolean model there are now almost no more points located. In particular this result means that for such a scenario basically two types of cells can be differed, "normal ones" like for the ordinary modulated Voronoi tessellation and slim ones that are covering the zones where no points are allowed. With regard to the estimated values for the cost functional defined in Section 4 the observation is that they increase as $\lambda_{1}$ tends to 0 (Fig. 9 (a)) for a fixed intensity $\lambda$ (here $\lambda=12$ ). Notice that for this example the intensities of the process $\tilde{X}$ are assumed to be equal, i.e., $\tilde{\lambda}_{1}=\tilde{\lambda}_{2}$. The 


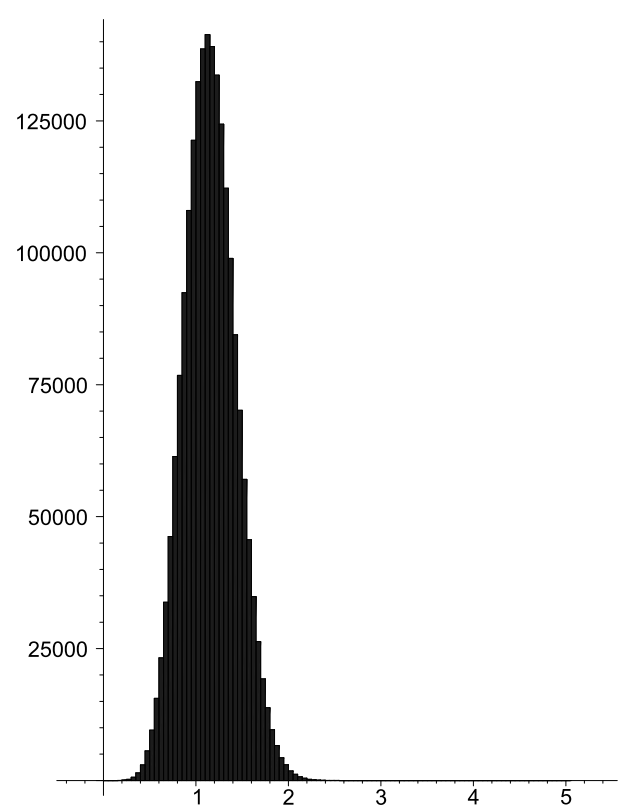

(a) $\lambda_{1}=12, \lambda_{2}=12$

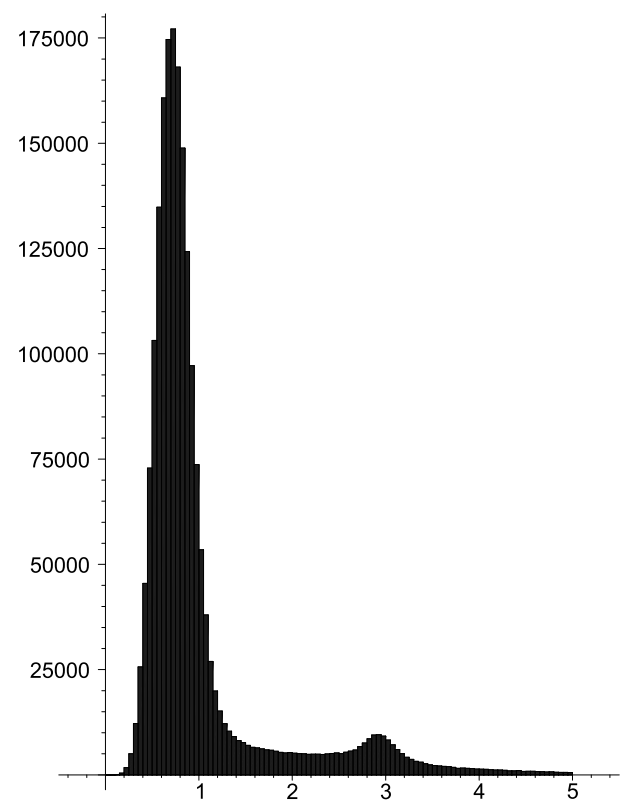

(c) $\lambda_{1}=0.05, \lambda_{2}=29.95$

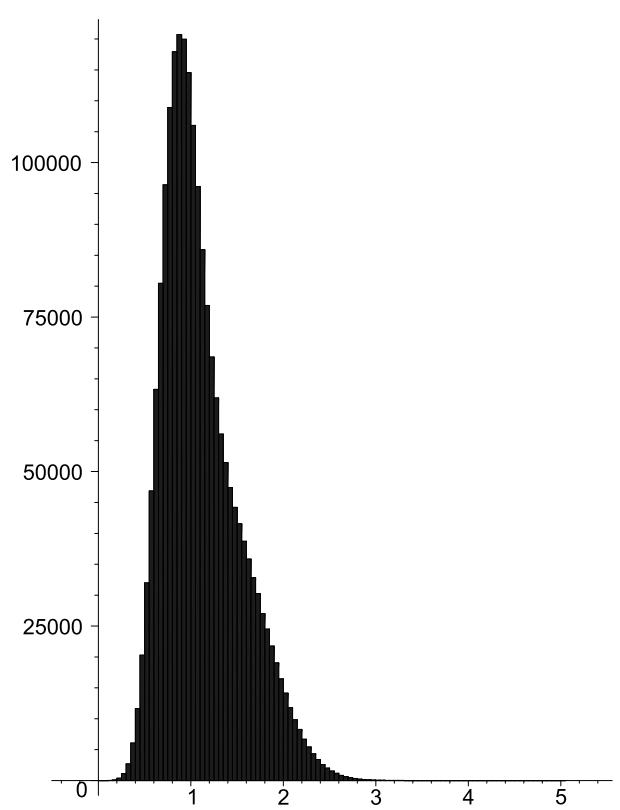

(b) $\lambda_{1}=6, \lambda_{2}=21$

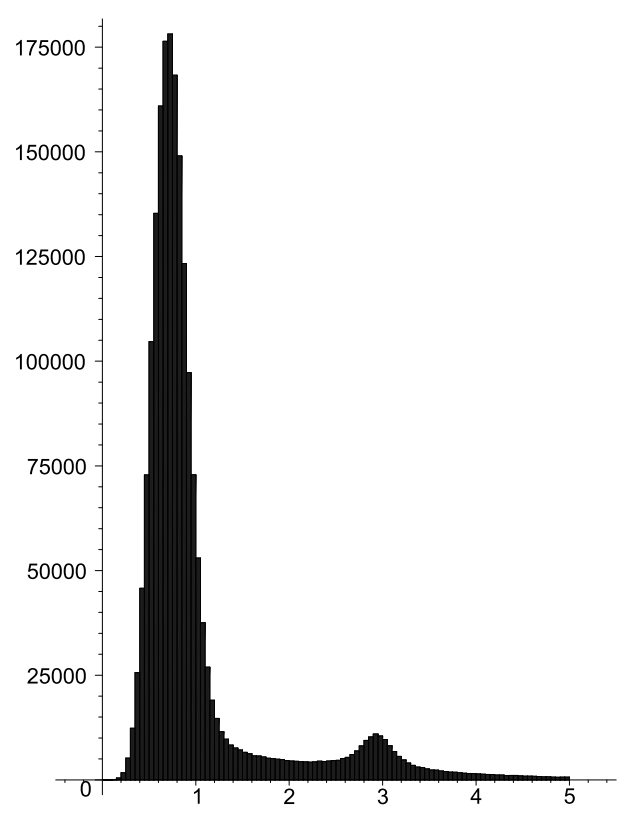

(d) $\lambda_{1}=0, \lambda_{2}=30$

Fig. 8. Perimeter of the typical cell 
sample size is again $n=2000000$ for each pair of parameters $\left(\lambda_{1}, \lambda_{2}\right)$. The effect of rising values for the cost functional if $\lambda_{1}$ tends to 0 can possibly be explained by the appearance of cells that have a relatively large ratio of perimeter to area. This causes a relatively large mean distance to the cell nuclei for points located in such cells. Notice that in the case of an ordinary Poisson-Voronoi tessellation $\left(\lambda_{1}=\lambda_{2}\right)$ the estimated value for the mean distance to the cell nuclei of 0.14437 coincides well with the theoretical value of $(2 \sqrt{\lambda})^{-1}=0.14434$.

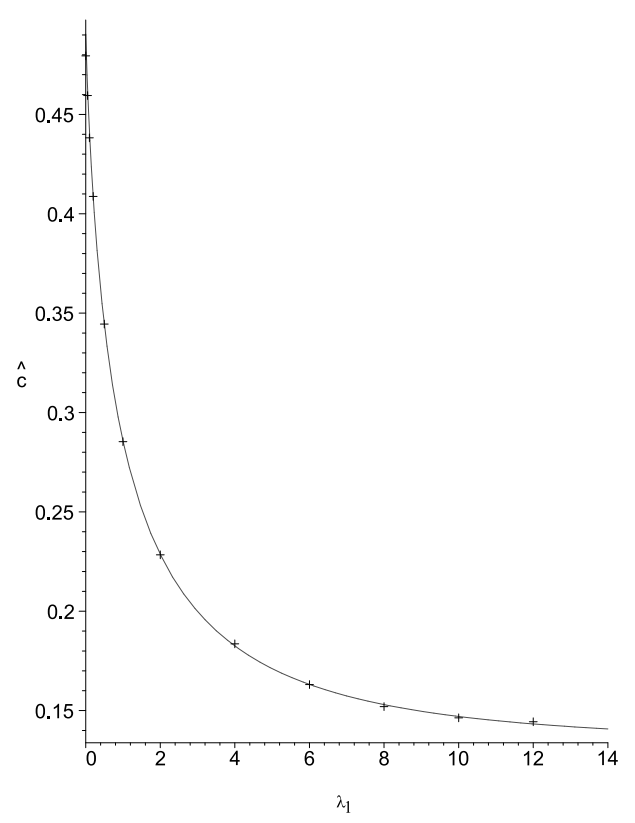

(a) $\lambda_{1}$ variable and $\tilde{\lambda}_{1}=\tilde{\lambda}_{2}$

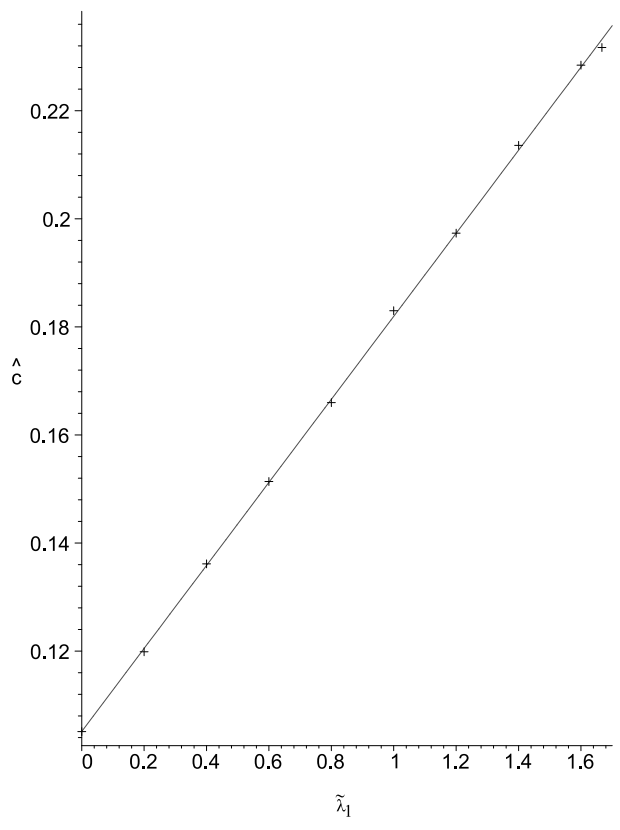

(b) $\lambda_{1}=4, \tilde{\lambda}=1$ and $\tilde{\lambda}_{1}$ variable

Fig. 9. Estimated cost functionals for $\lambda=12$ fixed

As a final numerical example we have a look at a scenario where $\tilde{\lambda}_{1} \neq \tilde{\lambda}_{2}$. For this scenario we take the same values for $\beta, p$ and $r$ as before and additionally keep $\lambda_{1}=4$ and $\lambda_{2}=24$ fixed. The values for $\tilde{\lambda}_{1}$ and $\tilde{\lambda}_{2}$ vary under the condition that $\tilde{\lambda}=1$. The results shown in Fig. 9 (b) display the linear relationship between the value of $\tilde{\lambda}_{1}$ and the estimated cost functional $\hat{c}$ which is a consequence of (4.5). Due to this linear relationship it suffices to estimate the two expectations $\mathbb{E}_{X}\left(\int_{\Xi_{X} \cap \Psi}|u| d u\right)$ and $\mathbb{E}_{X}\left(\int_{\Xi_{X} \cap \Psi^{c}}|u| d u\right)$ only for one specific pair of parameters $\tilde{\lambda}_{1}$ and $\tilde{\lambda}_{2}$ in order to obtain estimates of $c$ for all pairs of parameters $\tilde{\lambda}_{1}$ and $\tilde{\lambda}_{2}$ based on (4.5).

Notice that numerical evaluations of examples where $\tilde{\lambda}_{1} \neq \tilde{\lambda}_{2}$ are more time consuming due to the numerical computation of the estimator $\hat{c}$ introduced in (4.11) as opposed to the case where $\tilde{\lambda}_{1}=\tilde{\lambda}_{2}$ since here $\hat{c}$ is computed analytically, given realizations of the typical cell (Section 4.2). Therefore, for cases where $\hat{c}$ had to be computed numerically we took $n=100000$. 


\section{Discussion and outlook}

Big telecommunication operators need cost models and tools for strategic planning and pricing purposes. In order to be useful, these models must be able to reproduce the observed regional variability and to address relevant features from a telecommunication point of view. A detailed analysis of real data is often hard to achieve since it relies on huge databases and is sometimes confronted with the lack of data. Moreover such an analysis is in most cases only able to deliver observations but no explanations. Stochastic modeling is an alternative approach that directly provides a global view of the network together with clear assumptions on equipment locations and connections. In this context, the typical cell is a particularly important object since by its definition any result computed over repeated simulations of this cell can be compared to what is the result of measuring the whole tessellation that covers the area under study. The typical cell is thus representative for the global network behavior. For example, the area of the serving zone, i.e., the area of the typical cell of the tessellation, can be connected to the incoming demand for traffic via the number of customers. Various cost functionals and hence various components for pricing studies can be associated to the typical cell. It is then important to derive rapid and reliable algorithms for the simulation of the typical cell, allowing further integration of any functional depending on the geometric characteristics of the involved random processes and the typical cell. This paper focused on a basic example for such a functional which is the mean distance from a randomly chosen location within the cell to its corresponding cell nucleus (centre). All these characteristics and functionals can subsequently be combined and serve as components for more sophisticated cost models.

In this paper we presented an algorithm for the simulation of the typical cell for specific modulated Poisson-Voronoi tessellations, where the grains are generated by a Boolean model with circular grains of a fixed or random but bounded radius. Based on this algorithm we have shown how to efficiently estimate a basic functional, namely the mean distance from a random location to its Voronoi cell nucleus.

Extensions of the model can also be regarded. A natural way to achieve such an extended model would be, for example, to generate the modulation not by a single Boolean model $\Psi$ but by a sequence of Boolean models $\Psi_{1}, \ldots, \Psi_{n}$ with possibly different parameters with respect to the corresponding intensities and grain distribution. Another interesting extension would be to find a connection between the modulated Poisson-Voronoi tessellations that can be mainly used for modelling on a nationwide scale and models for urban access networks like the models provided by the Stochastic Subscriber Line Model (see Gloaguen, Coupé, Maier and Schmidt [10] and Gloaguen, Fleischer, Schmidt and Schmidt [11], [12]). This might enable an efficient analysis of other cost functionals like shortest path lengths along street systems or similar characteristics.

Apart from estimating the mean distance it is of a fundamental interest to estimate the distribution of the distance from a random location to its nearest Voronoi 
cell nucleus. In particular, such estimated distributions lead to the performance of risk analysis by looking at occurring tail probabilities.

Additionally other modulated Poisson-type tessellations like the modulated Poisson-Delaunay tessellation (Fig. 10) might be of interest. The modulated Poisson-Delaunay tessellation equipped with a realistic set of corresponding parameters might, for example, serve as a model for the connection between different towns. In general, not only for the case of modelling by modulated Poisson type tessellations, the question of determining realistic parameter values is a very complicated but also very rewardful one. Such a problem can of course only be solved by analyzing real data.

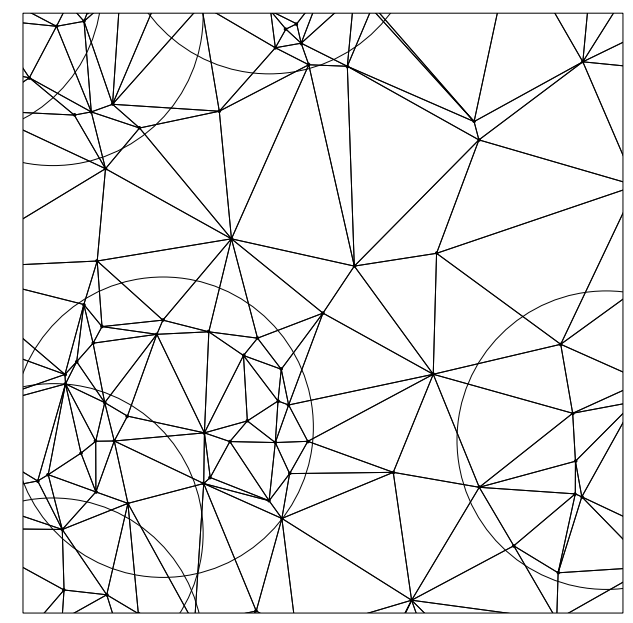

Fig. 10. A realization of a modulated Poisson-Delaunay tessellation

\section{Appendix A. Mathematical background}

In this appendix random (marked) point processes, Boolean models and random tessellations are defined and briefly explained. For a more detailed description and discussion of the topics mentioned the reader is referred to Schneider and Weil [17], and Stoyan, Kendall and Mecke [18], for example.

\section{A.1. Basic notations}

Let $\mathbb{R}$ and $\mathbb{N}$ be the set of real numbers and the set of positive integers, respectively; $\mathbb{N}_{0}=\mathbb{N} \cup\{0\}$. The abbreviations int $B, \partial B$, and $B^{c}$ are used to denote the interior, the boundary, and the complement of a set $B \subset \mathbb{R}^{2}$, respectively, where $\mathbb{R}^{2}$ denotes the 2-dimensional Euclidean space. For $x \in \mathbb{R}^{2}$ let $|x|$ denote the Euclidean norm of $x$. Furthermore, $B_{r}(x)$ and $B_{r}^{\neq}(x)$ denote, respectively, the 2-dimensional closed and open ball centered at $x \in \mathbb{R}^{2}$ with radius $r>0$, i.e., $B_{r}(x)=\left\{y \in \mathbb{R}^{2}:|x-y| \leq r\right\}$ and $B_{r}^{\neq}(x)=\left\{y \in \mathbb{R}^{2}:|x-y|<r\right\}$. By $\mathcal{B}\left(\mathbb{R}^{2}\right)$ the Borel $\sigma$-algebra on $\mathbb{R}^{2}$ is denoted. $\mathcal{B}_{0}\left(\mathbb{R}^{2}\right)$ is the family of bounded Borel sets in $\mathbb{R}^{2}$. 
On $\mathbb{R}^{2}$ we now define two topological groups, namely the group of all translations $t_{x}: y \mapsto y+x$ for $x \in \mathbb{R}^{2}$ and the group of all rotations $\vartheta_{R}: y \mapsto R y$ around the origin, where $R$ denotes a $2 \times 2$ matrix, orthogonal and with $\operatorname{det} R=1$. This allows us to introduce the following operations on sets $B \subset \mathbb{R}^{2}$, the translation $t_{x} B=\{y+x: y \in B\}$ for $x \in \mathbb{R}^{2}$ and the rotation $\vartheta_{R} B=\left\{\vartheta_{R} x: x \in B\right\}$ around the origin $o$, respectively.

Furthermore, introduce $\mathcal{F}, \mathcal{K}$, and $\mathcal{C}$ as the families of all closed sets, compact sets, and convex bodies (compact and convex sets) in $\mathbb{R}^{2}$, respectively. Recall that a random closed set $\Xi$ in $\mathbb{R}^{2}$ is a measurable mapping $\Xi: \Omega \rightarrow \mathcal{F}$ from some probability space $(\Omega, \mathcal{A}, \mathbb{P})$ into the measurable space $(\mathcal{F}, \mathcal{B}(\mathcal{F}))$, where $\mathcal{B}(\mathcal{F})$ denotes the smallest $\sigma$-algebra of subsets of $\mathcal{F}$ that contains all sets $\{F \in \mathcal{F}: F \cap K \neq \emptyset\}$ for any $K \in \mathcal{K}$. Particularly, the random closed set $\Xi$ is called a random compact set or a random convex body if $\mathbb{P}(\Xi \in \mathcal{K})=1$ or $\mathbb{P}(\Xi \in \mathcal{C})=1$, respectively.

Let $M$ be the set of simple and locally finite counting measures and let $\mathcal{M}$ be the smallest $\sigma$-algebra of subsets of $M$ that contains all sets of the form $\{\varphi \in M: \varphi(B)=j\}$, where $j \in \mathbb{N}_{0}$ and $B \in \mathcal{B}\left(\mathbb{R}^{2}\right)$. We introduce the shift operator $t_{x}: M \rightarrow M$ defined by $t_{x} \varphi(B)=\varphi\left(t_{x}^{-1} B\right)=\varphi\left(t_{-x} B\right)$ for $x \in \mathbb{R}^{2}$ as well as the rotation operator $\vartheta_{R}: M \rightarrow M$ by $\vartheta_{R} \varphi(B)=\varphi\left(\vartheta_{R}^{-1} B\right)=\varphi\left(\vartheta_{R^{-1}} B\right)$ for any rotation $R$ around the origin.

\section{A.2. Planar point processes}

A random point process $X$ in $\mathbb{R}^{2}$ is a measurable mapping $X: \Omega \rightarrow M$ from some probability space $(\Omega, \mathcal{A}, \mathbb{P})$ into the measurable space $(M, \mathcal{M})$. Therefore, a (simple) point process $X$ can be regarded as a counting measure $\sum_{x \in \operatorname{supp}(X)} \delta_{x}$ and $X(B)=\sum_{x \in \operatorname{supp}(X)} \delta_{x}(B)$ as the (random) number of points of $X$ in $B \in \mathcal{B}\left(\mathbb{R}^{2}\right)$. A point process $X$ can on the other hand be identified as a (planar) random closed set. In this case it is convenient to write $X=\left\{X_{n}\right\}_{n \geq 1}$, which expresses $X$ as a sequence $X_{1}, X_{2}, \ldots$ of random vectors $X_{n}: \Omega \rightarrow \mathbb{R}^{2}$ for $n \geq 1$ such that $\#\left\{n:\left|X_{n}\right|<r\right\}<$ $\infty$ for any $r>0$.

The distribution of $X$ is given as $P_{X}(A)=\mathbb{P}(X \in A)$ for $A \in \mathcal{M}$. The point process $X$ is called stationary if $P_{X}=P_{t_{x} X}$ for any $x \in \mathbb{R}^{2}$. It is called isotropic if $P_{X}=P_{\vartheta_{R} X}$ for any rotation $\vartheta_{R}$ around the origin.

The intensity measure $\Lambda: \mathcal{B}\left(\mathbb{R}^{2}\right) \rightarrow[0, \infty]$ of a point process $X$ is defined by

$$
\Lambda(B)=\mathbb{E} X(B), \quad B \in \mathcal{B}\left(\mathbb{R}^{2}\right) .
$$

If $X$ is stationary and $\Lambda$ is not equal to the zero measure we get that

$$
\Lambda(B)=\lambda|B|
$$

where $|B|$ denotes the Lebesgue measure of $B$, and where the constant $\lambda>0$ is called the intensity of $X$. A stationary point process $X$ with distribution $P_{X}$ is said to be ergodic if there are no other stationary point processes $X^{\prime}$ and $X^{\prime \prime}$ with distributions $P_{X^{\prime}}$ and $P_{X^{\prime \prime}}$, respectively, such that

$$
P_{X}=\alpha P_{X^{\prime}}+(1-\alpha) P_{X^{\prime \prime}}
$$


for an $\alpha \in(0,1)$. A geometric interpretation of ergodicity is that if we regard the set of distributions for stationary point processes as a simplex then the set of distributions for ergodic stationary point processes consists of the vertices of this simplex. Notice that analogous definitions of ergodicity exist for stationary random closed sets and stationary random tessellations.

Let now $\Lambda: \mathcal{B}\left(\mathbb{R}^{2}\right) \rightarrow[0, \infty]$ be any diffuse and locally finite measure on $\mathcal{B}\left(\mathbb{R}^{2}\right)$. A (simple) point process $X$ in $R^{2}$ that fulfills

$$
\mathbb{P}(X(B)=k)=e^{-\Lambda(B)} \frac{\Lambda(B)^{k}}{k !}, \quad B \in \mathcal{B}_{0}\left(\mathbb{R}^{2}\right), k \in \mathbb{N}_{0},
$$

is called a Poisson point process with intensity measure $\Lambda$ (see Fig. 3 (a) for a realization of a stationary Poisson point process). A possible generalization of a Poisson process is to take the measure $\Lambda$ itself random. This leads to Cox processes. If $P_{\Lambda}$ denotes the distribution of a Poisson process with intensity measure $\Lambda$, and $Q$ is the distribution of a random measure, then the point process $X$ with distribution $P_{X}: \mathcal{M} \rightarrow[0,1]$ given by

$$
P_{X}(A)=\int P_{\Lambda}(A) Q(d \Lambda), \quad A \in \mathcal{M}
$$

is called a Cox process. Hence we can think of a Cox process as a two-step random mechanism. In a first step a measure $\Lambda$ is determined according to a distribution $Q$. Afterwards, in a second step, a Poisson process is generated according to the intensity measure $\Lambda$.

\section{A.3. Planar marked point processes}

An extension of planar point processes can be achieved by additionally equipping the points with a mark taken from a mark space $D$. Mathematically, one assumes that $D$ represents a Polish space and denotes by $\mathcal{B}(D)$ the $\sigma$-algebra of its Borel sets. Regard $M_{D}=M\left(\mathbb{R}^{2} \times D\right)$, the set of all measures $\psi: \mathcal{B}\left(\mathbb{R}^{2}\right) \times \mathcal{B}(D) \rightarrow$ $\mathbb{N}_{0} \cup\{\infty\}$ that are simple and locally finite with respect to the first component. Furthermore, let $\mathcal{M}_{D}=\mathcal{M}\left(\mathbb{R}^{2} \times D\right)$ be the smallest $\sigma$-algebra of subsets of $M_{D}$ containing all sets of the form $\left\{\psi \in M_{D}: \psi(B \times G)=j\right\}$ for $B \in \mathcal{B}_{0}\left(\mathbb{R}^{2}\right), G \in \mathcal{B}(D)$ and $j \in \mathbb{N}_{0}$. Then a random marked point process $X_{D}$ is a mapping from $(\Omega, \mathcal{A}, \mathbb{P})$ into $\left(M_{D}, \mathcal{M}_{D}\right)$ with mark space $(D, \mathcal{B}(D))$.

The distribution $P_{X_{D}}$ of $X_{D}$ is given by $P_{X_{D}}(A)=\mathbb{P}\left(X_{D} \in A\right), A \in \mathcal{M}_{D}$. As for unmarked point processes an alternative representation of $X_{D}=\left\{\left[X_{n}, D_{n}\right]\right\}_{n \geq 1}$ as a collection of random marked points is often convenient. Then $X_{D}$ is said to be independently marked if $\left\{X_{n}\right\}_{n \geq 1}$ and $\left\{D_{n}\right\}_{n \geq 1}$ are independent and if $\left\{D_{n}\right\}_{n \geq 1}$ consists of independent and identically distributed random variables. Stationarity and isotropy for marked point processes are defined with respect to the first component, in other words the locations, of the marked point process. 
The intensity measure $\Lambda_{D}: \mathcal{B}\left(\mathbb{R}^{2}\right) \otimes \mathcal{B}(D) \rightarrow[0, \infty]$ of a marked point process $X_{D}$ is defined by

$$
\Lambda_{D}(B \times G)=\mathbb{E} X_{D}(B \times G), \quad B \in \mathcal{B}\left(\mathbb{R}^{2}\right), G \in \mathcal{B}(D) .
$$

Hence, $\Lambda_{D}(B \times G)$ is the expected number of points of $X_{D}$ in $B$ with a mark in $G$. If $X_{D}$ is stationary, the intensity measure $\Lambda_{D}$ can be decomposed as

$$
\Lambda_{D}(B \times G)=\lambda \int_{\mathbb{R}^{2}} \int_{D} \mathbb{1}_{B}(x) \mathbb{1}_{G}(m) P(d m) d x, \quad B \in \mathcal{B}\left(\mathbb{R}^{2}\right), G \in \mathcal{B}(D),
$$

where $\lambda>0$ is called intensity and $P: \mathcal{B}(D) \rightarrow[0,1]$ is the Palm mark distribution of $X_{D}$, given by

$$
P(G)=\frac{1}{\lambda|B|} \mathbb{E} \sum_{[x, m] \in \operatorname{supp}\left(X_{D}\right)} \mathbb{1}_{B}(x) \mathbb{1}_{G}(m), \quad G \in \mathcal{B}(D),
$$

for any $B \in \mathcal{B}\left(\mathbb{R}^{2}\right)$ with $0<|B|<\infty$.

\section{A.4. Neveu's exchange formula}

In the following Neveu's exchange formula for Palm distributions of (marked) point process in $\mathbb{R}^{2}$ is presented. This formula is useful in order to express the relationship of expectations of functionals of two stationary point processes with respect to their Palm distributions.

We consider a flow $\left\{\theta_{x}: x \in \mathbb{R}^{2}\right\}$ on the space $\Omega$, i.e., a family of bijective shift operators $\theta_{x}: \Omega \rightarrow \Omega$ such that $\theta_{x} \circ \theta_{y}=\theta_{x+y}$, where $\circ$ denotes the concatenation operator. Let us furthermore assume that the mapping $f: \mathbb{R}^{2} \times \Omega \rightarrow \Omega$ with $f(x, \omega)=\theta_{x} \omega$ is measurable. For $x \in \mathbb{R}^{2}$ we assume that $\theta_{x}$ is compatible with our shift operator $t_{x}$ as defined in Section A.1, which means that

$$
X_{D}\left(\theta_{x} \omega, B \times G\right)=t_{x} X_{D}(\omega, B \times G)=X_{D}\left(\omega, t_{-x} B \times G\right),
$$

for any marked point process $X_{D}: \Omega \rightarrow M_{D}$ and all $B \in \mathcal{B}\left(\mathbb{R}^{2}\right), G \in \mathcal{B}(D)$. Notice that then we can get the stationarity of $X_{D}$ by assuming that

$$
\mathbb{P}\left(\theta_{x} A\right)=\mathbb{P}\left(\theta_{x}^{-1} A\right)=\mathbb{P}(A), \quad A \in \mathcal{A} \text { and } x \in \mathbb{R}^{2},
$$

where $\theta_{x} A=\left\{\theta_{x} \omega: \omega \in A\right\}$.

Suppose that (A.9) holds. Then, we are able to introduce the Palm probability measure $\mathbb{P}_{X_{D}}^{*}$ for a stationary marked point process $X_{D}$ as the probability measure $\mathbb{P}_{X_{D}}^{*}$ on $\mathcal{A} \otimes \mathcal{B}(D)$ by

$$
\mathbb{P}_{X_{D}}^{*}(A \times G)=\frac{1}{\lambda|B|} \int_{\Omega} \int_{\mathbb{R}^{2} \times G} \mathbb{1}_{B}(x) \mathbb{1}_{A}\left(\theta_{x} \omega\right) X(\omega, d(x, g)) \mathbb{P}(d \omega)
$$

for any $B \in \mathcal{B}\left(\mathbb{R}^{2}\right)$ with $0<|B|<\infty$. 
Theorem A.1 (Neveu's exchange formula). Let $X_{D}$ and $\tilde{X}_{\tilde{D}}$ be arbitrary stationary marked point processes on $(\Omega, \mathcal{A}, \mathbb{P})$ with mark spaces $D$ and $\tilde{D}$ and intensities $\lambda$ and $\tilde{\lambda}$, respectively. Then, for any measurable function $f: \mathbb{R}^{2} \times D \times$ $\tilde{D} \times \Omega \rightarrow[0, \infty)$,

$$
\begin{aligned}
& \lambda \int_{\Omega \times D} \int_{\mathbb{R}^{2} \times \tilde{D}} f\left(x, g, \tilde{g}, \theta_{x} \omega\right) \tilde{X}_{\tilde{D}}(\omega, d(x, \tilde{g})) \mathbb{P}_{X_{D}}^{*}(d(\omega, g)) \\
& =\tilde{\lambda} \int_{\Omega \times \tilde{D}} \int_{\mathbb{R}^{2} \times D} f(-x, g, \tilde{g}, \omega) X_{D}(\omega, d(x, g)) \mathbb{P}_{\tilde{X}_{\tilde{D}}}^{*}(d(\omega, \tilde{g})) .
\end{aligned}
$$

\section{A.5. Boolean germ-grain model}

Consider a stationary Poisson point process $Y=\left\{Y_{n}\right\}$ with intensity $\beta>0$. Let $M_{1}, M_{2}, \ldots$ be a sequence of independent and identically distributed random closed sets in $\mathbb{R}^{2}$ with $\mathbb{E}\left|M_{0}\right|^{2}<\infty$, where $M_{0}$ is a generic representant of the sequence $\left\{M_{n}\right\}_{n \geq 1}$. Furthermore, let $\left\{Y_{n}\right\}$ and $\left\{M_{n}\right\}$ be independent. Then we call the random closed set

$$
\Psi=\bigcup_{n \geq 1}\left(Y_{n}+M_{n}\right)
$$

a Boolean germ-grain model. In Fig. 3 (b) a realization of a Boolean model is displayed. The point process $\left\{Y_{n}\right\}$ is called the germ process of $\Psi$ with germs $Y_{n}$, whereas the process $\left\{M_{n}\right\}$ is called the grain process of $\Psi$ with grains $M_{n}$. Throughout this paper only Boolean models with circular grains centered at the origin are regarded, where the radius of the circle can be either deterministic or random but bounded.

\section{A.6. Random tessellations}

A tessellation in $\mathbb{R}^{2}$ is a countable family $\tau=\left\{C_{n}\right\}_{n \geq 1}$ of convex bodies $C_{n} \in \mathcal{C}$ such that $\operatorname{int} C_{n} \neq \emptyset$ for all $n, \operatorname{int} C_{n} \cap \operatorname{int} C_{m}=\emptyset$ for all $n \neq m, \bigcup_{n \geq 1} C_{n}=\mathbb{R}^{2}$, and $\#\left\{n: C_{n} \cap K \neq \emptyset\right\}<\infty$ for any $K \in \mathcal{K}$. Notice that the sets $C_{n}$, called the cells of $\tau$, are polygons in $\mathbb{R}^{2}$. The family of all tessellations in $\mathbb{R}^{2}$ is denoted by $\mathcal{T}$. A random tessellation $\tau=\left\{\Xi_{n}\right\}_{n \geq 1}$ in $\mathbb{R}^{2}$ is a sequence of random convex bodies $\Xi_{n}$ such that $\mathbb{P}(\tau \in \mathcal{T})=1$. Notice that a random tessellation $\tau=\left\{\Xi_{n}\right\}_{n \geq 1}$ can also be considered as a marked point process $\sum_{n \geq 1} \delta_{\left[\alpha\left(\Xi_{n}\right), \Xi_{n}^{o}\right]}$, where $\alpha: \mathcal{C}^{\prime} \rightarrow \mathbb{R}^{2}$, $\mathcal{C}^{\prime}=\mathcal{C} \backslash\{\emptyset\}$, is a measurable mapping such that $\alpha(C) \in C$ and $\alpha(C+x)=\alpha(C)+x$ for any $C \in \mathcal{C}^{\prime}$ and $x \in \mathbb{R}^{d}$, and where $\Xi_{n}^{o}=\Xi_{n}-\alpha\left(\Xi_{n}\right)$ is the centered cell corresponding to $\Xi_{n}$ which contains the origin. The point $\alpha(C) \in \mathbb{R}^{2}$ is called an associated point of $C$ and can be chosen, for example, to be the lexicographically smallest point of $C$.

\section{A.7. Typical cell of stationary tessellations}

Suppose that the marked point process $X_{\tau}=\sum_{n \geq 1} \delta_{\left[\alpha\left(\Xi_{n}\right), \Xi_{n}^{o}\right]}$ is stationary with positive and finite intensity $\lambda_{\tau}=\mathbb{E} \#\left\{n: \alpha\left(\Xi_{n}\right) \in[0,1)^{2}\right\}$. By $\mathcal{P}^{o}$ we denote the family of all convex polytopes with their associated point at the origin. Then, the Palm mark distribution $P$ of $X_{\tau}$ is given by $P(B)=\lambda_{\tau}^{-1} \mathbb{E} \#\left\{n: \alpha\left(\Xi_{n}\right) \in[0,1)^{2}\right.$, 
$\left.\Xi_{n}^{o} \in B\right\}$ for any $B \in \mathcal{B}(\mathcal{F}) \cap \mathcal{P}^{o}$. Notice that a random polytope $\Xi^{*}: \Omega \rightarrow \mathcal{P}^{o}$, whose distribution coincides with $P$, is called the typical cell of $X_{\tau}$. Furthermore, it holds that

$$
\lambda_{\tau}^{-1}=\int_{\mathcal{P}^{o}}|C| P(d C),
$$

i.e., the expected area $\mathbb{E}\left|\Xi^{*}\right|=\int_{\mathcal{P} o}|C| P(d C)$ of the typical cell $\Xi^{*}$ is equal to $\lambda_{\tau}^{-1}$.

\section{A.8. Tessellations induced by point processes}

Let $S=\left\{x_{1}, x_{2}, \ldots\right\}$ be a locally finite set of points in $\mathbb{R}^{2}$. For $x_{n}, x_{m} \in S$ define the halfplane $H\left(x_{n}, x_{m}\right)$ by

$$
H\left(x_{n}, x_{m}\right)=\left\{x \in \mathbb{R}^{2}:\left|x-x_{n}\right| \leq\left|x-x_{m}\right|\right\} .
$$

Then we call the polygon $P_{n}$ given by

$$
P\left(x_{n}\right)=\bigcap_{m \neq n} H\left(p_{n}, p_{m}\right)=\left\{x \in \mathbb{R}^{2}:\left|x-x_{n}\right| \leq\left|x-x_{n}\right|, \forall m \neq n\right\} .
$$

the Voronoi cell of the point $x_{n}$. If we suppose that for each $x \in S$ we have that $P(x)$ is bounded, the sequence $\tau=\left\{P\left(x_{n}\right): x_{n} \in B\right\}$ is called the Voronoi tessellation with respect to $S$. The point $x_{n}$ is often denoted as the nucleus of $P\left(x_{n}\right)$. The Poisson-Voronoi tessellation can now be defined as the Voronoi tessellation $\tau_{X}$ that is induced by a Poisson point process $X=\left\{X_{1}, X_{2}, \ldots\right\}$.

Let $S=\left\{x_{1}, x_{2}, \ldots\right\}$ be a locally finite set of points $\mathbb{R}^{2}$ that is not collinear, i.e., if $x_{i}, x_{j}, x_{k}$ are three pairwise different points in $S$ it holds that there does not exist a line with the property that $x_{i}, x_{j}, x_{k}$ are all located on that line. Furthermore, let $\tau^{\prime}=\left\{P\left(x_{n}\right)\right\}$ be the Voronoi tessellation with respect to $S$. Let $Q=\left\{q_{1}, q_{2}, \ldots\right\}$ be the set of vertices of $\tau^{\prime}$ and $x_{i_{1}}, \ldots, x_{i_{k_{i}}}$ be the points in $S$ whose Voronoi cells share the vertex $q_{i}$. Let

$$
T_{i}=\left\{x \in \mathbb{R}^{2}: x=\sum_{j=1}^{k_{i}} \lambda_{j} x_{i_{j}}, \sum_{j=1}^{k_{i}} \lambda_{j}=1, \lambda_{j} \geq 0\right\}
$$

and let $\tau=\left\{T_{1}, \ldots, T_{m}\right\}$. Then, the set $\tau$ is called the Delaunay tessellation of $S$. Let $X=\left\{X_{1}, X_{2}, \ldots\right\}$ be a Poisson point process. The Delaunay tessellation induced by $X$ is called a Poisson-Delaunay tessellation.

Acknowledgements. This research was supported by France Telecom Research and Development through research grant No. 46132895 . The authors are grateful to K. Posch and A. Upowsky for their assistance in the simulations that led to the numerical results. The authors thank the referees for their valuable comments that led to an improvement of the manuscript. 


\section{References}

[1 ] F. Baccelli and B. Błaszczyszyn, On a coverage process ranging from the Boolean model to the Poisson-Voronoi tessellation. Advances in Applied Probability, 33 (2001), 293-323.

[ 2 ] F. Baccelli, C. Gloaguen and S. Zuyev, Superposition of Planar Voronoi Tessellations. Communications in Statistics, Series Stochastic Models, 16 (2000), 69-98.

[ 3 ] F. Baccelli, M. Klein, M. Lebourges and S. Zuyev, Géométrie aléatoire et architecture de réseaux. Annales des Télécommunication, 51 (1996), 158-179.

[ 4 ] F. Baccelli, M. Klein, M. Lebourges and S. Zuyev, Stochastic geometry and architecture of communication networks. Telecommunications Systems, 7 (1997), 209-227.

[ 5 ] F. Baccelli, D. Kofman and J.L. Rougier, Self organizing hierarchical multicast trees and their optimization. Proceedings of IEEE Infocom '99, New York, 1999, 1081-1089.

[6 ] F. Baccelli and S. Zuyev, Poisson-Voronoi spanning trees with applications to the optimization of communication networks. Operations Research, 47 (1996), 619-631.

[ 7 ] B. Błaszczyszyn and R. Schott, Approximate decomposition of some modulated PoissonVoronoi tessellations. Advances in Applied Probability, 35 (2003), 847-862.

[ 8 ] B. Błaszczyszyn and R. Schott, Approximations of functionals of some modulated PoissonVoronoi tessellations with applications to modeling of communication networks. Japan Journal of Industrial and Applied Mathematics, 22 (2004), 179-204.

[ 9 ] D.J. Daley and D. Vere-Jones, An Introduction to the Theory of Point Processes. Springer, New York, 1988.

[10] C. Gloaguen, P. Coupé, R. Maier and V. Schmidt, Stochastic modelling of urban access networks. Proc. 10th Internat. Telecommun. Network Strategy Planning Symp. (Munich, June 2002), VDE, Berlin, 2002, 99-104.

[11] C. Gloaguen, F. Fleischer, H. Schmidt and V. Schmidt, Simulation of typical Cox-Voronoi cells with a special regard to implementation tests. Mathematical Methods of Operations Research, 62 (2005), 357-373.

[12] C. Gloaguen, F. Fleischer, H. Schmidt and V. Schmidt, Analysis of shortest paths and subscriber line lengths in telecommunication access networks. Networks and Spatial Economics, 9 (2008), in print.

[13] R. Maier, J. Mayer and V. Schmidt, Distributional properties of the typical cell of stationary iterated tessellations. Mathematical Methods of Operations Research, 59 (2004), 287-302.

[14] J. Mayer and R. Guderlei, Test oracles and randomness. Lecture Notes in Informatics, P-58, Köllen Druck+Verlag GmbH, Bonn, 2004, 179-189.

[15] J. Mayer, V. Schmidt and F. Schweiggert, A unified simulation framework for spatial stochastic models. Simulation Modelling Practice and Theory, 12 (2004), 307-326.

[16] M.P. Quine and D.F. Watson, Radial generation of $n$-dimensional Poisson processes. Journal of Applied Probability, 21 (1984), 548-557.

[17] R. Schneider and W. Weil, Stochastische Geometrie. Teubner, Stuttgart, 2000.

[18] D. Stoyan, W.S. Kendall and J. Mecke, Stochastic Geometry and Its Applications, 2nd edition. J. Wiley \& Sons, Chichester, 1995.

[19] K. Tchoumatchenko and S. Zuyev, Aggregate and fractal tessellations. Probability Theory Related Fields, 121 (2001), 198-218.

[20] J.G. Wendel, A problem in geometric probability. Math. Scand., 11 (1962), 109-111. 